\title{
Self-consistent RPA and the time-dependent density matrix approach $^{\star}$
}

\author{
P. Schuck ${ }^{1,2, a}$ and M. Tohyama ${ }^{3}$ \\ 1 Institut de Physique Nucléaire, F-91406 Orsay CEDEX, France \\ 2 Laboratoire de Physique et Modélisation des Milieux Condensés, CNRS and Université Joseph Fourier, 25 Avenue des Martyrs \\ BP166, F-38042 Grenoble Cédex 9, France \\ 3 Kyorin University School of Medicine, Mitaka, Tokyo 181-8611, Japan
}

Received: 4 May 2016 / Revised: 18 August 2016

Published online: 11 October 2016 - C Società Italiana di Fisica / Springer-Verlag 2016

Communicated by N. Alamanos

\begin{abstract}
The time-dependent density matrix (TDDM) or BBGKY (Bogoliubov, Born, Green, Kirkwood, Yvon) approach is decoupled and closed at the three-body level in finding a natural representation of the latter in terms of a quadratic form of two-body correlation functions. In the small amplitude limit an extended RPA coupled to an also extended second RPA is obtained. Since including two-body correlations means that the ground state cannot be a Hartree-Fock state, naturally the corresponding RPA is upgraded to Self-Consistent RPA (SCRPA) which was introduced independently earlier and which is built on a correlated ground state. SCRPA conserves all the properties of standard RPA. Applications to the exactly solvable Lipkin and the 1D Hubbard models show good performances of SCRPA and TDDM.
\end{abstract}

\section{Introduction}

Approaches to many-body physics are many-fold. Only on the mean-field level there is consensus in practically all fields of physics. On the level of two-body correlations, the approaches diverge. There are the Quantum Monte Carlo methods with a reference state (usually a Slater determinant or a BCS state) on which a local twobody operator in the exponential is applied $[1,2]$ which will have some relation with the Self-Consistent RPA (SCRPA) approach we will present here. There is the Coupled Cluster Theory (CCT) [3,4] most directly applicable for ground-state energies. The Density Matrix Renormalisation Group (DRMG) method is very successful mostly in 1D systems [5-7]. The correlated basis functions method is still another many-body theory often applied to nuclear matter [8]. Many more attempts to tackle with the difficult many-body problem, all taylored to specific problems, could be cited. In such a diversity, it may be worth presenting recent progress with the equation of motion method where the hierarchy of time-dependent density matrices

\footnotetext{
* Contribution to the Topical Issue "Finite range effective interactions and associated many-body methods - A tribute to Daniel Gogny" edited by Nicolas Alamanos, Marc Dupuis, Nathalie Pillet.

a e-mail: schuck@ipno.in2p3.fr
}

is truncated at the three-body level in approximating the latter by a quadratic form of two-body correlation functions meeting in this way SCRPA as a sub-product of the theory. These equations are attractive in the sense that they are of the Schroedinger type still keeping all appreciated properties of standard RPA, i.e., conservation laws and sum rules are fulfilled. In the case of broken symmetries the Goldstone mode appears, Ward identities and gauge invariance are maintained. Ground-state energies and excited states are obtained on the same footing. We will demonstrate these properties with applications to model cases. We are sure that Daniel Gogny would have been very interested in this approach, since he had a deep knowledge in many-body theory.

This short review is organised as follows. In sect. 2, we will explain how we truncate and close the system of density matrix equations expressing the three-body correlator by a quadratic form of the two-body correlator. In sect. 3, we consider the small amplitude limit of those equations. In sect. 4 we give a reminder of Self-Consistent RPA (SCRPA) and in sect. 5, the relation of SCRPA with the Coupled Cluster Doubles (CCD) wave function [9] is outlined and it will be explained how to obtain the occupation numbers. In sect. 6 , we present applications to the Lipkin and Hubbard models and in sect. 7 we give our conclusion. 


\section{Truncation of the time-dependent density matrix equations}

\subsection{General formalism}

We start from the two-body Hamiltonian for fermions in second quantisation,

$$
H=\sum_{\alpha} e_{\alpha} a_{\alpha}^{+} a_{\alpha}+\frac{1}{4} \sum_{\alpha \beta \gamma \delta} \bar{v}_{\alpha \beta \gamma \delta} a_{\alpha}^{+} a_{\beta}^{+} a_{\delta} a_{\gamma},
$$

where $e_{\alpha}$ are some single-particle energies, $a^{+}, a$ are the usual fermion creation and annihilation operators, and $\bar{v}_{\alpha \beta \gamma \delta}=\langle\alpha \beta|v| \gamma \delta\rangle-\langle\alpha \beta|v| \delta \gamma\rangle$ is the antisymmetrized matrix element of the two-body interaction.

The coupled equations for the one-body and two-body density matrices are then of the following form [10]:

$$
\begin{aligned}
i \dot{\rho}_{\alpha \alpha^{\prime}}= & \sum_{\lambda}\left(\epsilon_{\alpha \lambda} \rho_{\lambda \alpha^{\prime}}-\rho_{\alpha \lambda} \epsilon_{\lambda \alpha^{\prime}}\right) \\
& +\frac{1}{2} \sum_{\lambda_{1} \lambda_{2} \lambda_{3}}\left[\bar{v}_{\alpha \lambda_{1} \lambda_{2} \lambda_{3}} C_{\lambda_{2} \lambda_{3} \alpha^{\prime} \lambda_{1}}\right. \\
& \left.-C_{\alpha \lambda_{1} \lambda_{2} \lambda_{3}} \bar{v}_{\lambda_{2} \lambda_{3} \alpha^{\prime} \lambda_{1}}\right] \\
i \dot{C}_{\alpha \beta \alpha^{\prime} \beta^{\prime}}= & \sum_{\lambda}\left(\epsilon_{\alpha \lambda} C_{\lambda \beta \alpha^{\prime} \beta^{\prime}}+\epsilon_{\beta \lambda} C_{\alpha \lambda \alpha^{\prime} \beta^{\prime}}\right. \\
& \left.-\epsilon_{\lambda \alpha^{\prime}} C_{\alpha \beta \lambda \beta^{\prime}}-\epsilon_{\lambda \beta^{\prime}} C_{\alpha \beta \alpha^{\prime} \lambda}\right) \\
& +B_{\alpha \beta \alpha^{\prime} \beta^{\prime}}^{0}+P_{\alpha \beta \alpha^{\prime} \beta^{\prime}}^{0}+H_{\alpha \beta \alpha^{\prime} \beta^{\prime}}^{0} \\
& +\frac{1}{2} \sum_{\lambda_{1} \lambda_{2} \lambda_{3}}\left[\bar{v}_{\alpha \lambda_{1} \lambda_{2} \lambda_{3}} C_{\lambda_{2} \lambda_{3} \beta \alpha^{\prime} \lambda_{1} \beta^{\prime}}\right. \\
& +\bar{v}_{\lambda_{1} \beta \lambda_{2} \lambda_{3}} C_{\lambda_{2} \lambda_{3} \alpha \alpha^{\prime} \lambda_{1} \beta^{\prime}} \\
& -\bar{v}_{\lambda_{1} \lambda_{2} \alpha^{\prime} \lambda_{3}} C_{\alpha \lambda_{3} \beta \lambda_{1} \lambda_{2} \beta^{\prime}} \\
& \left.-\bar{v}_{\lambda_{1} \lambda_{2} \lambda_{3} \beta^{\prime}} C_{\alpha \lambda_{3} \beta \lambda_{1} \lambda_{2} \alpha^{\prime}}\right]
\end{aligned}
$$

where $C_{\alpha \beta \alpha^{\prime} \beta^{\prime}}$ is the correlated part of the two-body density matrix, that is

$$
\rho_{\alpha \beta \alpha^{\prime} \beta^{\prime}}=\mathcal{A}\left(\rho_{\alpha \alpha^{\prime}} \rho_{\beta \beta^{\prime}}\right)+C_{\alpha \beta \alpha^{\prime} \beta^{\prime}}
$$

and $C_{3}$ (in short-hand notation) contained in the threebody density matrix

$$
\begin{aligned}
\rho_{\alpha \beta \gamma, \alpha^{\prime} \beta^{\prime} \gamma^{\prime}}= & \mathcal{A S}\left(\rho_{\alpha \alpha^{\prime}} \rho_{\beta \beta^{\prime}} \rho_{\gamma \gamma^{\prime}}+\rho_{\alpha \alpha^{\prime}} C_{\beta \gamma \beta^{\prime} \gamma^{\prime}}\right) \\
& +C_{\alpha \beta \gamma, \alpha^{\prime} \beta^{\prime} \gamma^{\prime}}
\end{aligned}
$$

is the fully correlated part of the three-body density matrix in (5) which is neglected in the original version of TDDM [11]. In (4) and (5), $\mathcal{A}$ and $\mathcal{S}$ are the appropriate antisymmetrisers and symmetrisers. The energy (meanfield) matrix $\epsilon_{\alpha \alpha^{\prime}}$ is given by

$$
\epsilon_{\alpha \alpha^{\prime}}=e_{\alpha} \delta_{\alpha \alpha^{\prime}}+\sum_{\lambda_{1} \lambda_{2}} \bar{v}_{\alpha \lambda_{1} \alpha^{\prime} \lambda_{2}} \rho_{\lambda_{2} \lambda_{1}} .
$$

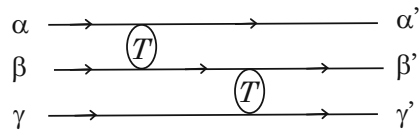

(a)

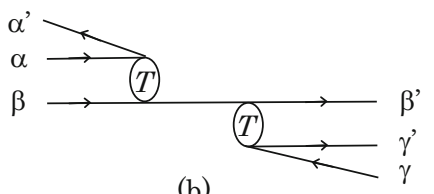

Fig. 1. Second-order $T$-matrix contribution to the Faddeev series of the three-body correlation function.

The matrix $B_{\alpha \beta \alpha^{\prime} \beta^{\prime}}^{0}$ in eq. (3) does not contain $C_{\alpha \beta \alpha^{\prime} \beta^{\prime}}$ and describes the $2 p-2 h$ and $2 h-2 p$ excitations,

$$
\begin{aligned}
B_{\alpha \beta \alpha^{\prime} \beta^{\prime}}^{0}= & \sum_{\lambda_{1} \lambda_{2} \lambda_{3} \lambda_{4}} \bar{v}_{\lambda_{1} \lambda_{2} \lambda_{3} \lambda_{4}} \\
& \times\left[\left(\delta_{\alpha \lambda_{1}}-\rho_{\alpha \lambda_{1}}\right)\left(\delta_{\beta \lambda_{2}}-\rho_{\beta \lambda_{2}}\right) \rho_{\lambda_{3} \alpha^{\prime}} \rho_{\lambda_{4} \beta^{\prime}}\right. \\
& \left.-\rho_{\alpha \lambda_{1}} \rho_{\beta \lambda_{2}}\left(\delta_{\lambda_{3} \alpha^{\prime}}-\rho_{\lambda_{3} \alpha^{\prime}}\right)\left(\delta_{\lambda_{4} \beta^{\prime}}-\rho_{\lambda_{4} \beta^{\prime}}\right)\right] .
\end{aligned}
$$

Particle-particle $(p-p)$ and hole-hole $(h-h)$ correlations are taken care of by $P_{\alpha \beta \alpha^{\prime} \beta^{\prime}}^{0}$,

$$
\begin{aligned}
P_{\alpha \beta \alpha^{\prime} \beta^{\prime}}^{0}= & \frac{1}{2} \sum_{\lambda_{1} \lambda_{2} \lambda_{3} \lambda_{4}} \bar{v}_{\lambda_{1} \lambda_{2} \lambda_{3} \lambda_{4}} \\
& \times\left[\left(\delta_{\alpha \lambda_{1}} \delta_{\beta \lambda_{2}}-\delta_{\alpha \lambda_{1}} \rho_{\beta \lambda_{2}}-\rho_{\alpha \lambda_{1}} \delta_{\beta \lambda_{2}}\right) C_{\lambda_{3} \lambda_{4} \alpha^{\prime} \beta^{\prime}}\right. \\
& \left.-\left(\delta_{\lambda_{3} \alpha^{\prime}} \delta_{\lambda_{4} \beta^{\prime}}-\delta_{\lambda_{3} \alpha^{\prime}} \rho_{\lambda_{4} \beta^{\prime}}-\rho_{\lambda_{3} \alpha^{\prime}} \delta_{\lambda_{4} \beta^{\prime}}\right) C_{\alpha \beta \lambda_{1} \lambda_{2}}\right] .
\end{aligned}
$$

$H_{\alpha \beta \alpha^{\prime} \beta^{\prime}}^{0}$ contains the $p$ - $h$ correlations.

$$
\begin{aligned}
H_{\alpha \beta \alpha^{\prime} \beta^{\prime}}^{0}= & \sum_{\lambda_{1} \lambda_{2} \lambda_{3} \lambda_{4}} \bar{v}_{\lambda_{1} \lambda_{2} \lambda_{3} \lambda_{4}} \\
& \times\left[\delta_{\alpha \lambda_{1}}\left(\rho_{\lambda_{3} \alpha^{\prime}} C_{\lambda_{4} \beta \lambda_{2} \beta^{\prime}}-\rho_{\lambda_{3} \beta^{\prime}} C_{\lambda_{4} \beta \lambda_{2} \alpha^{\prime}}\right)\right. \\
& +\delta_{\beta \lambda_{2}}\left(\rho_{\lambda_{4} \beta^{\prime}} C_{\lambda_{3} \alpha \lambda_{1} \alpha^{\prime}}-\rho_{\lambda_{4} \alpha^{\prime}} C_{\lambda_{3} \alpha \lambda_{1} \beta^{\prime}}\right) \\
& -\delta_{\alpha^{\prime} \lambda_{3}}\left(\rho_{\alpha \lambda_{1}} C_{\lambda_{4} \beta \lambda_{2} \beta^{\prime}}-\rho_{\beta \lambda_{1}} C_{\lambda_{4} \alpha \lambda_{2} \beta^{\prime}}\right) \\
& \left.-\delta_{\beta^{\prime} \lambda_{4}}\left(\rho_{\beta \lambda_{2}} C_{\lambda_{3} \alpha \lambda_{1} \alpha^{\prime}}-n_{\alpha \lambda_{2}} C_{\lambda_{3} \beta \lambda_{1} \alpha^{\prime}}\right)\right] .
\end{aligned}
$$

In eq. (3), the last four terms contain the correlated part $C_{3}$ of the three-body density matrix. In the past, usually $\mathrm{C}_{3}$ was neglected. We here want to keep it, approximating it by a quadratic form of $C_{2}$ 's which sums up an important sub-class of correlations contained in $C_{3}$. Inspecting (3), we see that the $C_{3}$ 's have $a^{+} a a$ attached to the interaction. Therefore, the $C_{3}$ 's are of the 2 particle- 1 hole $(2 p$ $1 h)$ or $2 h-1 p$ type. Such type of correlations enter, e.g., the single-particle self-energy of the Dyson equation [12]. An important subclass of Feynman diagrams in $C_{3}$ are the one-line reducible ones where the $2 p-1 h$ states collapse into $1 p$ ones (as this is, e.g., the case in first-order perturbation theory). This class of correlations we want to incorporate in our approach. They are graphically represented by a 
second-order $T$-matrix approach of the three-body Faddeev series in fig. 1.

This quadratic form of the three-body correlation matrix is most systematically and straightforwardly obtained from the identity between three-body and four-body density matrices

$$
\rho_{\alpha \beta \gamma \alpha^{\prime} \beta^{\prime} \gamma^{\prime}}=\frac{1}{N-3} \sum_{\lambda} \rho_{\alpha \beta \gamma \lambda \alpha^{\prime} \beta^{\prime} \gamma^{\prime} \lambda .} .
$$

The above identity is written in terms of correlation matrices as

$$
\begin{aligned}
C_{\alpha \beta \gamma \alpha^{\prime} \beta^{\prime} \gamma^{\prime}}= & \frac{1}{3} \sum_{\lambda}\left(\rho_{\alpha \lambda} C_{\lambda \beta \gamma \alpha^{\prime} \beta^{\prime} \gamma^{\prime}}+\rho_{\beta \lambda} C_{\alpha \lambda \gamma \alpha^{\prime} \beta^{\prime} \gamma^{\prime}}\right. \\
& +\rho_{\gamma \lambda} C_{\alpha \beta \lambda \alpha^{\prime} \beta^{\prime} \gamma^{\prime}}+\rho_{\lambda \alpha^{\prime}} C_{\alpha \beta \gamma \lambda \beta^{\prime} \gamma^{\prime}} \\
& +\rho_{\lambda \beta^{\prime}} C_{\alpha \beta \gamma \alpha^{\prime} \lambda \gamma^{\prime}}+\rho_{\lambda \gamma^{\prime}} C_{\alpha \beta \gamma \alpha^{\prime} \beta^{\prime} \lambda} \\
& -C_{\alpha \beta \alpha^{\prime} \lambda} C_{\gamma \lambda \beta^{\prime} \gamma^{\prime}}-C_{\alpha \beta \gamma^{\prime} \lambda} C_{\gamma \lambda \alpha^{\prime} \beta^{\prime}} \\
& -C_{\alpha \beta \lambda \beta^{\prime}} C_{\gamma \lambda \alpha^{\prime} \gamma^{\prime}}-C_{\alpha \gamma \alpha^{\prime} \lambda} C_{\lambda \beta \beta^{\prime} \gamma^{\prime}} \\
& -C_{\alpha \gamma \beta^{\prime} \lambda} C_{\beta \lambda \alpha^{\prime} \gamma^{\prime}}-C_{\alpha \gamma \lambda \gamma^{\prime}} C_{\beta \lambda \alpha^{\prime} \beta^{\prime}} \\
& -C_{\alpha \lambda \alpha^{\prime} \beta^{\prime}} C_{\beta \gamma \gamma^{\prime} \lambda}-C_{\alpha \lambda \alpha^{\prime} \gamma^{\prime}} C_{\beta \gamma \lambda \beta^{\prime}} \\
& \left.-C_{\alpha \lambda \beta^{\prime} \gamma^{\prime}} C_{\beta \gamma \alpha^{\prime} \lambda}-C_{\alpha \beta \gamma \lambda \alpha^{\prime} \beta^{\prime} \gamma^{\prime} \lambda}\right),
\end{aligned}
$$

where $C_{\alpha \beta \gamma \lambda \alpha^{\prime} \beta^{\prime} \gamma^{\prime} \lambda}$ is a four-body correlation matrix. Under the assumptions that $\rho_{\alpha \alpha^{\prime}}=\delta_{\alpha \alpha^{\prime}} n_{\alpha}$ and $C_{\alpha \beta \gamma \lambda \alpha^{\prime} \beta^{\prime} \gamma^{\prime} \lambda}=0$, that is the fully correlated part, $C_{4}$, is neglected, the above relation is given as

$$
\begin{aligned}
C_{\alpha \beta \gamma \alpha^{\prime} \beta^{\prime} \gamma^{\prime}}= & \frac{1}{3-n_{\alpha}-n_{\beta}-n_{\gamma}-n_{\alpha^{\prime}}-n_{\beta^{\prime}}-n_{\gamma^{\prime}}} \\
& \times \sum_{\lambda}\left(-C_{\alpha \beta \alpha^{\prime} \lambda} C_{\gamma \lambda \beta^{\prime} \gamma^{\prime}}-C_{\alpha \beta \gamma^{\prime} \lambda} C_{\gamma \lambda \alpha^{\prime} \beta^{\prime}}\right. \\
& -C_{\alpha \beta \lambda \beta^{\prime}} C_{\gamma \lambda \alpha^{\prime} \gamma^{\prime}}-C_{\alpha \gamma \alpha^{\prime} \lambda} C_{\lambda \beta \beta^{\prime} \gamma^{\prime}} \\
& -C_{\alpha \gamma \beta^{\prime} \lambda} C_{\beta \lambda \alpha^{\prime} \gamma^{\prime}}-C_{\alpha \gamma \lambda \gamma^{\prime}} C_{\beta \lambda \alpha^{\prime} \beta^{\prime}} \\
& -C_{\alpha \lambda \alpha^{\prime} \beta^{\prime}} C_{\beta \gamma \gamma^{\prime} \lambda}-C_{\alpha \lambda \alpha^{\prime} \gamma^{\prime}} C_{\beta \gamma \lambda \beta^{\prime}} \\
& \left.-C_{\alpha \lambda \beta^{\prime} \gamma^{\prime}} C_{\beta \gamma \alpha^{\prime} \lambda}\right) .
\end{aligned}
$$

The correlated part of the occupation numbers in (12) may induce correlations of four-body type which we want to neglect. Therefore, we will replace in (12) the $n_{\alpha}$ by their Hartree-Fock (HF) values zero or one. In principle the system of equations is now closed and, given initial conditions, one could start with a time-dependent solution. However, also a static-equilibrium solution and small-amplitude solutions around equilibrium are of particular interest. We want to elaborate those cases next.

\subsection{Static limit: restriction to particles and holes}

In the static limit, the first equation (2) becomes a generalised HF equation (6) where the single-particle basis couples back to the ground-state correlations

$$
\begin{aligned}
0= & \sum_{\lambda}\left(\epsilon_{\alpha \lambda} \rho_{\lambda \alpha^{\prime}}-\rho_{\alpha \lambda} \epsilon_{\lambda \alpha^{\prime}}\right) \\
& +\frac{1}{2} \sum_{\lambda_{1} \lambda_{2} \lambda_{3}}\left[\bar{v}_{\alpha \lambda_{1} \lambda_{2} \lambda_{3}} C_{\lambda_{2} \lambda_{3} \alpha^{\prime} \lambda_{1}}\right. \\
& \left.-C_{\alpha \lambda_{1} \lambda_{2} \lambda_{3}} \bar{v}_{\lambda_{2} \lambda_{3} \alpha^{\prime} \lambda_{1}}\right]
\end{aligned}
$$

Such equation has been considered in the past [13]. The static equation for the two-body correlation function, for convenience, is written in the basis where the mean-field energies (6) are diagonal,

$$
\begin{aligned}
0= & \left(\epsilon_{\alpha}+\epsilon_{\beta}-\epsilon_{\alpha^{\prime}}-\epsilon_{\beta^{\prime}}\right) C_{\alpha \beta \alpha^{\prime} \beta^{\prime}}+B_{\alpha \beta \alpha^{\prime} \beta^{\prime}}^{0}+P_{\alpha \beta \alpha^{\prime} \beta^{\prime}}^{0} \\
& +H_{\alpha \beta \alpha^{\prime} \beta^{\prime}}^{0}+T_{\alpha \beta \alpha^{\prime} \beta^{\prime}} .
\end{aligned}
$$

As above the three-body part is given by

$$
\begin{aligned}
& T_{\alpha \beta \alpha^{\prime} \beta^{\prime}}= \\
& \frac{1}{2} \sum_{\lambda_{1} \lambda_{2} \lambda_{3}}\left[\bar{v}_{\alpha \lambda_{1} \lambda_{2} \lambda_{3}} C_{\lambda_{2} \lambda_{3} \beta \alpha^{\prime} \lambda_{1} \beta^{\prime}}+\bar{v}_{\lambda_{1} \beta \lambda_{2} \lambda_{3}} C_{\lambda_{2} \lambda_{3} \alpha \alpha^{\prime} \lambda_{1} \beta^{\prime}}\right. \\
& \left.-\bar{v}_{\lambda_{1} \lambda_{2} \alpha^{\prime} \lambda_{3}} C_{\alpha \lambda_{3} \beta \lambda_{1} \lambda_{2} \beta^{\prime}}-\bar{v}_{\lambda_{1} \lambda_{2} \lambda_{3} \beta^{\prime}} C_{\alpha \lambda_{3} \beta \lambda_{1} \lambda_{2} \alpha^{\prime}}\right]
\end{aligned}
$$

Again, approximating the $C_{3}$ 's by the quadratic form (12) yields a closed set of equations. However, it may be useful to solve those equations in the (generalised) HF basis which defines particle $(p)$ states above the Fermi sea and hole $(h)$ states below. One easily imagines then that correlation functions with an odd number of $p(h)$-indices are suppressed with respect to those with an even number. We, therefore, will only consider the following four index combinations of the $C_{2}$ 's:

$$
C_{p p^{\prime} h h^{\prime}} ; C_{p_{1} h_{1} p_{2} h_{2}} ; C_{p_{1} p_{2} p_{3} p_{4}} ; C_{h_{1} h_{2} h_{3} h_{4}} .
$$

That those combinations are the most important ones, may also be warranted by the fact that the $2 p$ - $2 h$ states are the only ones which enter the $B^{0}$ matrix in the $\mathrm{HF}$ limit implying that among the four correlation functions, again $C_{p p^{\prime} h h^{\prime}}$ is the most important one. Among the threebody correlation functions the following are the dominant ones (as long as one is not close to a macroscopic phase transition or to systems with a Goldstone (zero) mode) (please note that in the following the denominator of (12) is equal to -1$)$ :

$$
C_{p_{1} h_{1} h_{2}, p_{2} h_{3} h_{4}} \simeq \sum_{p} C_{p_{1} p h_{3} h_{4}} C_{h_{1} h_{2} p_{2} p}
$$

that is the product of two correlation functions with $2 p-2 h$ indices is the most important one as argued above. There exists only one further 3-body correlation function which has this specific product property

$$
C_{h_{1} p_{1} p_{2}, h_{2} p_{3} p_{4}} \simeq \sum_{h} C_{p_{1} p_{2} h_{2} h} C_{h h_{1} p_{3} p_{4}}
$$

Respecting this approximation scheme, we obtain for the four possible three-body terms

$$
\begin{aligned}
& T_{p_{1} p_{2} h_{1} h_{2}}= \\
& \frac{1}{2} \sum_{p p^{\prime} h h^{\prime}}\left[\bar{v}_{p_{1} p h h^{\prime}} C_{p_{2} p^{\prime} h_{1} h_{2}} C_{h h^{\prime} p p^{\prime}}-\left(p_{1} \leftrightarrow p_{2}\right)\right] \\
& +\frac{1}{2} \sum_{h h^{\prime} p p^{\prime}}\left[\bar{v}_{p p^{\prime} h_{1} h} C_{p_{1} p_{2} h_{2} h^{\prime}} C_{h^{\prime} h p p^{\prime}}-\left(h_{1} \leftrightarrow h_{2}\right)\right],
\end{aligned}
$$




$$
\begin{aligned}
T_{p_{1} h_{1} p_{2} h_{2}}= & \frac{1}{2} \sum_{p p^{\prime} h h^{\prime}} \bar{v}_{p_{1} h h^{\prime} p} C_{p^{\prime} p h_{2} h} C_{h_{1} h^{\prime} p_{2} p^{\prime}} \\
& +\frac{1}{2} \sum_{p p^{\prime} p^{\prime \prime} h} \bar{v}_{p_{1} p p^{\prime} p^{\prime \prime}} C_{p^{\prime} p^{\prime \prime} h h_{2}} C_{h_{1} h p_{2} p} \\
& -\frac{1}{2} \sum_{p p^{\prime} h h^{\prime}} \bar{v}_{p h^{\prime} p_{2} h} C_{h h_{1} p p^{\prime}} C_{p_{1} p^{\prime} h^{\prime} h_{2}} \\
& -\frac{1}{2} \sum_{p p^{\prime} p^{\prime \prime} h} \bar{v}_{p^{\prime} p^{\prime \prime} p_{2} p} C_{p_{1} p h_{2} h} C_{h h_{1} p^{\prime} p^{\prime \prime}} \\
& -\frac{1}{2} \sum_{p p^{\prime} h h^{\prime}} \bar{v}_{h_{1} p p^{\prime} h} C_{h^{\prime} h p_{2} p} C_{p^{\prime} p_{1} h^{\prime} h_{2}} \\
& +\frac{1}{2} \sum_{p h h^{\prime} h^{\prime \prime}} \bar{v}_{h_{1} h h^{\prime} h^{\prime \prime}} C_{h^{\prime} h^{\prime \prime} p_{2} p} C_{p p_{1} h h_{2}} \\
& +\frac{1}{2} \sum_{p p^{\prime} h h^{\prime}} \bar{v}_{p h^{\prime} h_{2} p^{\prime}} C_{p_{1} p^{\prime} h h^{\prime}} C_{h_{1} h p_{2} p} \\
& -\frac{1}{2} \sum_{p h h^{\prime} h^{\prime \prime}} \bar{v}_{h^{\prime} h^{\prime \prime} h h_{2}} C_{p p_{1} h^{\prime} h^{\prime \prime}} C_{h_{1} h p_{2} p} .
\end{aligned}
$$

The exchange matrix $T_{h_{1} p_{1} p_{2} h_{2}}$ of $T_{p_{1} h_{1} p_{2} h_{2}}$ is given not by changing $p_{1}$ and $h_{1}$ on the right-hand side of eq. (19) but by using eqs. (15), (16) and (17). Then the exchange property $T_{h_{1} p_{1} p_{2} h_{2}}=-T_{p_{1} h_{1} p_{2} h_{2}}$ is satisfied. Furthermore, we have

$$
\begin{aligned}
& T_{p_{1} p_{2} p_{3} p_{4}}= \\
& \frac{1}{2} \sum_{p h h^{\prime} h^{\prime \prime}}\left[\bar{v}_{p_{1} h p h^{\prime}} C_{p p_{2} h h^{\prime \prime}} C_{h^{\prime \prime} h^{\prime} p_{3} p_{4}}-\left(p_{1} \leftrightarrow p_{2}\right)\right] \\
& -\frac{1}{2} \sum_{p h h^{\prime} h^{\prime \prime}}\left[\bar{v}_{p h^{\prime} p_{3} h} C_{p_{1} p_{2} h h^{\prime \prime}} C_{h^{\prime \prime} h^{\prime} p p_{4}}-\left(p_{3} \leftrightarrow p_{4}\right)\right], \\
& T_{h_{1} h_{2} h_{3} h_{4}}= \\
& \frac{1}{2} \sum_{p p^{\prime} p^{\prime \prime} h}\left[\bar{v}_{h_{1} p p^{\prime} h} C_{h h_{2} p^{\prime \prime} p} C_{p^{\prime} p^{\prime \prime} h_{3} h_{4}}-\left(h_{1} \leftrightarrow h_{2}\right)\right] \\
& -\frac{1}{2} \sum_{p p^{\prime} p^{\prime \prime} h}\left[\bar{v}_{p^{\prime} h h_{3} p} C_{h_{1} h_{2} p^{\prime \prime} p^{\prime}} C_{p p^{\prime \prime} h h_{4}}-\left(h_{3} \leftrightarrow h_{4}\right)\right] .
\end{aligned}
$$

For the numerical solution of those static correlation functions several possibilities exist. They are explained in [14]. The coupled set of equations for the four $C_{2}$ 's will be used in the TDDM applications presented below.

The above coupled equations have a number of appealing properties. They are totally antisymmetric and they are number and energy conserving.

We next want to elaborate on the small amplitude limit of TDDM.

\section{The small amplitude limit}

\subsection{Derivation of STDDM* with non-linear terms}

Time-dependent Hartree-Fock (TDHF) leads in the small amplitude limit, as is well known, to RPA with exchange (we will keep the acronym RPA including exchange as this is used in nuclear physics). Therefore, the corresponding ground state is a Slater determinant. Including two-body correlations, the ground state cannot be a Slater determinant any longer. It must also contain correlations. The small amplitude limit of TDDM will lead to an eigenvalue problem connecting the one-body sector with the two-body one. It will be interesting to see in which way the one-body sector will be modified with respect to RPA including the ground-state correlations.

Let us, therefore, take the small amplitude limit of our coupled equations derived above. With

$$
\rho_{1}=\rho_{1}^{(0)}+\delta \rho_{1}, \quad C_{2}=C_{2}^{(0)}+\delta C_{2}
$$

and

$$
\begin{aligned}
\delta \rho_{1} & =\sum_{\nu}\left[\tilde{\chi}^{\nu} e^{-i \Omega_{\nu} t}+\tilde{\chi}^{\nu,+} e^{i \Omega_{\nu} t}\right] \\
\delta C_{2} & =\sum_{\nu}\left[\tilde{\mathcal{X}}^{\nu} e^{-i \Omega_{\nu} t}+\tilde{\mathcal{X}}^{\nu,+} e^{i \Omega_{\nu} t}\right]
\end{aligned}
$$

we obtain coupled equations for the one-body and twobody transition amplitudes $\tilde{\chi}_{\alpha \alpha^{\prime}}^{\nu}=\left\langle\nu\left|: a_{\alpha^{\prime}}^{+} a_{\alpha}:\right| 0\right\rangle$ and $\tilde{\mathcal{X}}_{\alpha \beta \alpha^{\prime} \beta^{\prime}}^{\nu}=\left\langle\nu\left|: a_{\alpha^{\prime}}^{+} a_{\beta^{\prime}}^{+} a_{\beta} a_{\alpha}:\right| 0\right\rangle:$

$$
\left(\begin{array}{cc}
a & b \\
c & \tilde{d}
\end{array}\right)\left(\begin{array}{c}
\tilde{\chi}^{\nu} \\
\tilde{\mathcal{X}}^{\nu}
\end{array}\right)=\Omega_{\nu}\left(\begin{array}{c}
\tilde{\chi}^{\nu} \\
\tilde{\mathcal{X}}^{\nu}
\end{array}\right) .
$$

where : $a_{\lambda}^{+} a_{\lambda^{\prime}}:=a_{\lambda}^{+} a_{\lambda^{\prime}}-\rho_{\lambda \lambda^{\prime}}$ and : $a_{\lambda_{1}}^{+} a_{\lambda_{2}}^{+} a_{\lambda_{2}^{\prime}} a_{\lambda_{1}^{\prime}}:=$ $a_{\lambda_{1}}^{+} a_{\lambda_{2}}^{+} a_{\lambda_{2}^{\prime}} a_{\lambda_{1}^{\prime}}-\left[\rho_{\lambda_{1} \lambda_{1}^{\prime}} \rho_{\lambda_{2} \lambda_{2}^{\prime}}-\rho_{\lambda_{1} \lambda_{2}^{\prime}} \rho_{\lambda_{2} \lambda_{1}^{\prime}}\right]$. The matrix $\tilde{d}$ is written as $\tilde{d}=d+\Delta d$ where $d$ stems from the variation of the linear terms of the two-body correlation matrix whereas $\Delta d$ comes from the variation of the threebody correlation matrix when it is approximated as, e.g., in eq. (16) and (17) by quadratic forms of $C_{2}$ 's (that is, the leading contributions). The matrices $c$ and $\Delta d$ include the two-body correlation matrix. The matrices in eq. (22) are given in appendix A. Equations (22) with $\Delta d=0$ have been called in the past STDDM (small TDDM) equations [15]. With the inclusion of the non-linear term $\Delta d$, we want to call those STDDM* equations.

Inspection of $a, b$ matrices tells us that the matrix in (22) is highly non-symmetric. This stems from the fact that the amplitudes $\tilde{\chi}$ and $\tilde{\mathcal{X}}$ are linearly dependent. In the next section, we will clarify this point and introduce a rotation of the vector which makes the corresponding matrix essentially symmetric.

\subsection{STDDM and STDDM* from an Extended Second RPA (ESRPA): relation with SCRPA}

In this section we will make a connection with eq. (22) coming from a seemingly quite different side of attack. Let us, therefore, consider the Equation of Motion (EOM) approach [16] with one- and two-body sectors included without restriction of indices. We, thus, define the following 
generalised RPA operator:

$$
\mathcal{Q}_{\nu}^{+}=\sum\left[\chi_{\lambda \lambda^{\prime}}^{\nu}: a_{\lambda}^{+} a_{\lambda^{\prime}}:+\mathcal{X}_{\lambda_{1} \lambda_{2} \lambda_{1}^{\prime} \lambda_{2}^{\prime}}^{\nu}: a_{\lambda_{1}}^{+} a_{\lambda_{2}}^{+} a_{\lambda_{2}^{\prime}} a_{\lambda_{1}^{\prime}}:\right] .
$$

As usual with EOM for such an ansatz, we suppose

$$
\mathcal{Q}_{\nu}^{+}|0\rangle=|\nu\rangle \quad \text { and } \quad \mathcal{Q}_{\nu}|0\rangle=0 .
$$

Minimising the corresponding energy weighted sum rule $2 \Omega_{\nu}=\left\langle 0\left|\left[\mathcal{Q}_{\nu},\left[H, \mathcal{Q}_{\nu}{ }^{+}\right]\right]\right| 0\right\rangle /\left\langle 0\left|\left[\mathcal{Q}, \mathcal{Q}^{+}\right]\right| 0\right\rangle$, see sect. 4, we obtain the following eigenvalue problem:

$$
\left(\begin{array}{ll}
\mathcal{S} & \mathcal{B} \\
\mathcal{C} & \mathcal{D}
\end{array}\right)\left(\begin{array}{l}
\chi \\
\mathcal{X}
\end{array}\right)=\Omega\left(\begin{array}{cc}
\mathcal{N}_{1} & \mathcal{T} \\
\mathcal{T}^{+} & \mathcal{N}_{2}
\end{array}\right)\left(\begin{array}{l}
\chi \\
\mathcal{X}
\end{array}\right)
$$

where the various matrix elements are given in an obvious way by the corresponding double commutators (lhs) and commutators (rhs) which correspond to the ones contained in the sum rule for $\Omega_{\nu}$. The one-body sector $\mathcal{S} \chi=\Omega \mathcal{N}_{1} \chi$ will be given explicitly below in sect. 4 .

The matrices in eq. (24) are given in [17] where this equation was named ERPA (Extended RPA). However, a more appropriate name is "Extended Second RPA" (ESRPA) because it includes the two-body sector and reduces to the standard second RPA in the limit where the expectation values are evaluated with the $\mathrm{HF}$ state. It has been shown in the past that, under certain approximations, this ESRPA is equivalent to the STDDM equation [18]. Let us sketch this again. For this, in ESRPA, we neglect everywhere $C_{3}$ (and $\left.C_{4}\right)$. This concerns $\mathcal{B}, \mathcal{C}, \mathcal{D}$, and $\mathcal{N}_{2}$. In $\mathcal{D}$ we additionally neglect the terms which are named in [19] the $\mathcal{T}_{32}$ terms. Those $\mathcal{T}_{32}$ terms correspond to the expectation values of the commutator between two-body and three-body operators [19]. Then we arrive at the following structure of the above eigenvalue equation (24):

$$
\left(\begin{array}{l}
a \mathcal{N}_{1}+b \mathcal{T}^{+} a \mathcal{T}+b \mathcal{N}_{2} \\
c \mathcal{N}_{1}+d \mathcal{T}^{+} c \mathcal{T}+d \mathcal{N}_{2}
\end{array}\right)\left(\begin{array}{l}
\chi \\
\mathcal{X}
\end{array}\right)=\Omega\left(\begin{array}{cc}
\mathcal{N}_{1} & \mathcal{T} \\
\mathcal{T}^{+} & \mathcal{N}_{2}
\end{array}\right)\left(\begin{array}{l}
\chi \\
\mathcal{X}
\end{array}\right)
$$

where the matrices $a, b, c, d$ are as in (22) (see appendix A) containing at most $C_{2}$ 's.

Equation (25) is intimately related to the STDDM equation as we will show now. Defining

$$
\left(\begin{array}{c}
\tilde{\chi} \\
\tilde{\mathcal{X}}
\end{array}\right)=\left(\begin{array}{cc}
\mathcal{N}_{1} & \mathcal{T} \\
\mathcal{T}^{+} & \mathcal{N}_{2}
\end{array}\right)\left(\begin{array}{l}
\chi \\
\mathcal{X}
\end{array}\right)
$$

we obtain the following modified eigenvalue equation:

$$
\left(\begin{array}{ll}
a & b \\
c & d
\end{array}\right)\left(\begin{array}{l}
\tilde{\chi} \\
\tilde{\mathcal{X}}
\end{array}\right)=\Omega\left(\begin{array}{l}
\tilde{\chi} \\
\tilde{\mathcal{X}}
\end{array}\right)
$$

The remarkable fact is that this equation is also obtained in linearising around equilibrium the coupled EOM's for $n_{\alpha}$ and $C_{2}$ as is seen from eq. (22) without $\Delta d$. With the use of eq. (26) the STDDM* equation eq. (22) with $\Delta d$ included can also be expressed as

$$
\left(\begin{array}{l}
a \mathcal{N}_{1}+b \mathcal{T}+a \mathcal{T}+b \mathcal{N}_{2} \\
c \mathcal{N}_{1}+\tilde{d} \mathcal{T}+c \mathcal{T}+\tilde{d} \mathcal{N}_{2}
\end{array}\right)\left(\begin{array}{l}
\chi \\
\mathcal{X}
\end{array}\right)=\Omega\left(\begin{array}{cc}
\mathcal{N}_{1} & \mathcal{T} \\
\mathcal{T}^{+} & \mathcal{N}_{2}
\end{array}\right)\left(\begin{array}{l}
\chi \\
\mathcal{X}
\end{array}\right)
$$

Notice that with respect to $(25)$ the matrix $d$ is changed into $\tilde{d}$ in (28). With respect to (22), we want to call the set of equations (28), the STDDM*-b equations (or STDDM-b when $\Delta d$ is neglected). Since $\mathcal{T}, \mathcal{N}_{2}$ and $\Delta d$ contain $C_{2}$, the [21] and [22] elements of eq. (28) have additional quadratic terms of $C_{2}$ that correspond to $C_{3}$. Thus, STDDM*-b is, in principle, a better approximation to ESRPA than STDDM.

Let us remark that in the left matrix the elements [12] and [21] are Hermitian conjugates to one another. This stems from the fact that already in $(24)$ the matrices $\mathcal{C}$ and $\mathcal{B}$ are the Hermitian conjugates of one another under the condition that they are evaluated at equilibrium, see $[18,20]$ for a discussion of this point. The [11] element of the left matrix is also symmetric because at equilibrium we have $i \dot{\rho}=0$. The one-body sector of eq. (25) corresponds to Self-Consistent RPA (SCRPA), see below, which was derived independently earlier [16]. So, in including correlations, the standard RPA has been upgraded to SCRPA. This is natural because, as mentioned, with correlations the corresponding ground state cannot be the HF state any longer. Therefore SCRPA has now found its natural place when the time-dependent HF equations are extended in a consistent way to include two-body correlations. We will come back to SCRPA in sect. 4 and sect. 5 . The [22] element is not Hermitian because at this level of our theory we do not fulfill that 3-body and 4-body density matrices are stationary.

As already mentioned, in the above STDDM equation $b \neq c^{+}$and thus the corresponding matrix is strongly non-symmetric. One, therefore, has to define left and right eigenvectors. How this goes in detail is explained in [18] where also applications with good success are presented. On the other hand, (25) and (28) are much more symmetric versions of STDDM and STDDM*. The remaining non-Hermiticity in the [22] element of the interaction matrix in STDDM* may be eliminated by the prescription of Rowe [21,22] who explicitly symmetrised the matrix. If the two versions (27) and (25) of STDDM (STDDM*, if $\Delta d$ is included as in (28)) are solved in full, the results will be the same. However, the fact of transforming the non-symmetric form of STDDM in (27) to the more symmetric STDDM one in (25) has apparently transferred a lot of correlations from the two-body sector to the onebody sector (standard RPA vs. SCRPA). This may be of importance if in STDDM (or in $\mathrm{STDDM}^{*}$ ) further approximations are applied. An extreme approximation is to neglect the 2-body amplitudes in both cases where the difference clearly shows up. On the other hand, a non-Hermitian eigenvalue problem may also entail some problems concerning spurious solutions or non-positive definite spectral functions. However, in the past applications $[18,23,24]$, this has never caused any serious problems. In a way, the situation is rather similar to the difference which exists between the Dyson boson expansion which leads to a non-Hermitian problem and, e.g., the Holstein-Primakoff (Belyaev-Zelevinsky) boson expansion leading to a Hermitian matrix [12]. The basic difference between both methods is, as here, the treatment of the norm matrix. 
In summary of the present section, we can say that the linearisation of the TDDM equations has lead to a type of extended second RPA (ESRPA) equation which we named STDDM*-b which contains an important part of the threebody correlations (contained in the $\Delta d$ matrix). The onebody part of this equation is equivalent to what is known as SCRPA in the literature. It also contains already a good part of the three-body correlations as shown in [14]. The structure of SCRPA is not affected by the inclusion or not of $\Delta d$. So, SCRPA is already contained in STDDM-b, that is, without inclusion of the three-body correlations. SCRPA takes into account a correlated ground state as it should be in a theory which goes beyond TDHF. Before elaborating on SCRPA in the next sections, let us shortly explain how to claculate occupation numbers and correlation energies from ESRPA, STDDM-b, or STDDM*-b.

\subsection{Occupation numbers and correlation energy}

We shall now consider how the occupation probabilities $n_{\alpha}$ are expressed by the transition amplitudes in ESRPA, STDDM-b, or STDDM*-b. We assume the following relation for the diagonal occupation matrix $\rho_{\alpha \alpha^{\prime}}=n_{\alpha} \delta_{\alpha \alpha^{\prime}}$ :

$$
\begin{aligned}
\sum_{\nu} \tilde{\chi}_{\alpha \alpha^{\prime}}^{\nu} \tilde{\chi}_{\beta^{\prime} \beta}^{\nu *} & =\sum_{\nu}\left\langle 0\left|: a_{\alpha^{\prime}}^{+} a_{\alpha}:\right| \nu\right\rangle\left\langle\nu\left|: a_{\beta^{\prime}}^{+} a_{\beta}:\right| 0\right\rangle \\
& =\delta_{\alpha \beta^{\prime}}\left\langle 0\left|a_{\alpha^{\prime}}^{+} a_{\beta}\right| 0\right\rangle+\left\langle 0\left|: a_{\alpha^{\prime}}^{+} a_{\beta^{\prime}}^{+} a_{\beta} a_{\alpha}:\right| 0\right\rangle \\
& =\delta_{\alpha \beta^{\prime}} \delta_{\beta \alpha^{\prime}} n_{\beta} \bar{n}_{\alpha}+C_{\alpha \beta \alpha^{\prime} \beta^{\prime}}
\end{aligned}
$$

where $\bar{n}_{\alpha}=1-n_{\alpha}$. From eq. (29) we obtain

$$
\sum_{\nu} \tilde{\chi}_{\alpha \alpha}^{\nu} \tilde{\chi}_{\alpha \alpha}^{\nu *}=n_{\alpha}\left(1-n_{\alpha}\right)+C_{\alpha \alpha \alpha \alpha}=n_{\alpha}-n_{\alpha}^{2} .
$$

The above equation gives for the occupation numbers

$$
n_{\alpha}=\frac{1}{2}\left(1 \pm \sqrt{1-4 \sum_{\nu \neq 0} \tilde{\chi}_{\alpha \alpha}^{\nu} \tilde{\chi}_{\alpha \alpha}^{\nu *}}\right) .
$$

In RPA and SCRPA there is no diagonal one-body amplitude such as $\chi_{\alpha \alpha}^{\nu}$, whereas in ESRPA, STDDM-b, or $\mathrm{STDDM}^{*}$-b $\chi_{\alpha \alpha}^{\nu}$ can couple to $\mathcal{X}_{\alpha \beta \alpha^{\prime} \beta^{\prime}}^{\nu}$ which has the same quantum numbers as the ground state. Thus the occupation probabilities in ESRPA, STDDM-b, or STDDM*$\mathrm{b}$ are determined by two-phonon states expressed by $\mathcal{X}_{\alpha \beta \alpha^{\prime} \beta^{\prime}}^{\nu}$, which is in contrast with SCRPA. We use eq. (31) to calculate the occupation probabilities in ESRPA, etc. Let us notice that relation (31) has the same structure as the occupation numbers obtained from the BCS theory when expressed via the BCS amplitudes $v_{i} u_{i}=\kappa_{i}$ [12].

The correlation energy is usually defined as the difference of the total correlated energy minus the Hartree-Fock energy. In this work, we thought it more appropriate to consider what one could call the 2-body correlation energy (for example in the case of BCS theory, this would reduce to the pairing energy) $E_{2 \text { bcor }}$ defined by

$$
E_{2 \mathrm{bcor}}=\frac{1}{4} \sum_{\alpha \beta \alpha^{\prime} \beta^{\prime}} \bar{v}_{\alpha \beta \alpha^{\prime} \beta^{\prime}} C_{\alpha^{\prime} \beta^{\prime} \alpha \beta} .
$$

The equation for $\tilde{\chi}_{\alpha \alpha^{\prime}}^{\nu}$ in STDDM, $a \tilde{\chi}^{\nu}+b \tilde{\mathcal{X}}^{\nu}=\Omega_{\nu} \tilde{\chi}^{\nu}$, gives

$$
\begin{aligned}
\Omega_{\nu} \tilde{\chi}_{\alpha \alpha^{\prime}}^{\nu}= & \left(\epsilon_{\alpha}-\epsilon_{\alpha^{\prime}}\right) \tilde{\chi}_{\alpha \alpha^{\prime}}^{\nu} \\
& +\left(n_{\alpha^{\prime}}-n_{\alpha}\right) \sum_{\lambda \lambda^{\prime}} \bar{v}_{\alpha \lambda^{\prime} \alpha^{\prime} \lambda} \tilde{\chi}_{\lambda \lambda^{\prime}}^{\nu} \\
& +\frac{1}{2} \sum_{\lambda_{1} \lambda_{2} \lambda_{3}}\left(\bar{v}_{\alpha \lambda_{1} \lambda_{2} \lambda_{3}} \tilde{\mathcal{X}}_{\lambda_{2} \lambda_{3} \alpha^{\prime} \lambda_{1}}^{\nu}\right. \\
& \left.-\bar{v}_{\lambda_{1} \lambda_{2} \alpha^{\prime} \lambda_{3}} \tilde{\mathcal{X}}_{\alpha \lambda_{3} \lambda_{1} \lambda_{2}}^{\nu}\right) .
\end{aligned}
$$

Multiplying by $\tilde{\chi}_{\beta^{\prime} \beta}^{\nu *}$ and using

$$
\begin{aligned}
\sum_{\nu} \tilde{\mathcal{X}}_{\alpha \beta \alpha^{\prime} \beta^{\prime}}^{\nu} \tilde{\chi}_{\gamma^{\prime} \gamma}^{\nu *}= & \delta_{\alpha \gamma^{\prime}} C_{\gamma \beta \alpha^{\prime} \beta^{\prime}}-\delta_{\beta \gamma^{\prime}} C_{\gamma \alpha \alpha^{\prime} \beta^{\prime}} \\
& +n_{\gamma \alpha^{\prime}} C_{\alpha \beta \beta^{\prime} \gamma^{\prime}}-n_{\gamma \beta^{\prime}} C_{\alpha \beta \alpha^{\prime} \gamma^{\prime}} \\
& -n_{\beta \gamma^{\prime}} C_{\alpha \gamma \alpha^{\prime} \beta^{\prime}}+n_{\alpha \gamma^{\prime}} C_{\beta \gamma \alpha^{\prime} \beta^{\prime}} \\
& +C_{\alpha \beta \gamma \alpha^{\prime} \beta^{\prime} \gamma^{\prime}}
\end{aligned}
$$

and (29), we obtain

$$
\begin{aligned}
\sum_{\mu \alpha} \Omega_{\nu} \tilde{\chi}_{\alpha \alpha}^{\nu} \tilde{\chi}_{\alpha \alpha}^{\nu *} & =\sum_{\alpha \lambda \lambda^{\prime}} \bar{v}_{\alpha \lambda \alpha \lambda^{\prime}} C_{\alpha \lambda^{\prime} \alpha \lambda} \\
& -\frac{1}{2} \sum_{\alpha \lambda \lambda^{\prime} \lambda^{\prime \prime}} \bar{v}_{\lambda \lambda^{\prime} \alpha \lambda^{\prime \prime}} C_{\alpha \lambda^{\prime \prime} \lambda \lambda^{\prime}}
\end{aligned}
$$

The first term on the right-hand side has no contribution in the solvable models discussed below. In general, $C_{p h p h^{\prime}}$, $C_{p p^{\prime} p p^{\prime \prime}}, C_{h p h p^{\prime}}$ and $C_{h h^{\prime} h h^{\prime \prime}}$ are smaller than $C_{p p^{\prime} h h^{\prime}}$ and $C_{h h^{\prime} p p^{\prime}}$ in a perturbative regime. Therefore, $E_{2 \text { bcor }}$ can approximately be expressed as

$$
E_{2 \mathrm{bcor}} \approx-\frac{1}{2} \sum_{\nu \alpha} \Omega_{\nu} \tilde{\chi}_{\alpha \alpha}^{\nu} \tilde{\chi}_{\alpha \alpha}^{\nu *}
$$

Equation (36) has only diagonal elements $\tilde{\chi}_{\alpha \alpha}^{\nu}$, which means that in ESRPA $E_{2 \text { bcor }}$ is determined by two-phonon states similarly to the occupation probabilities (eq. (31)). We calculate $E_{2 \text { bcor }}$ in ESRPA, etc., using eq. (36) in the applications below. It will also be the expression we use for the applications in sect. 6 . Since with (31) we have the occupation numbers, we can also calculate the one-body part of the energy and, thus, the total energy is given as well.

\section{Self-consistent RPA}

\subsection{General outline}

As we have mentioned, the one-body sector of STDDM-b and $\mathrm{STDDM}^{*}$-b is equivalent to what is known in the literature as SCRPA. Because the one-body sector is of importance for applications but also in its own right, we, for completeness, will again dwell on it in this and the next section.

Let us start writing down the most general singleparticle RPA operator as

$$
Q_{\nu}^{+}=\sum_{\alpha \beta, \alpha \neq \beta} \chi_{\alpha \beta}^{\nu}: a_{\alpha}^{+} a_{\beta}:
$$


where, as usual,

$$
|\nu\rangle=Q_{\nu}^{+}|0\rangle
$$

is the excited state. The RPA operator also is supposed to possess the killing property (see sect. 5)

$$
Q_{\nu}|0\rangle=0
$$

We can define an average excitation energy using the energy weighted sum rule

$$
\Omega_{\nu}=\frac{1}{2} \frac{\left\langle 0\left|\left[Q_{\nu},\left[H, Q_{\nu}^{+}\right]\right]\right| 0\right\rangle}{\left\langle 0\left|\left[Q_{\nu}, Q_{\nu}^{+}\right]\right| 0\right\rangle} .
$$

Varying $\Omega_{\nu}$ with respect to the amplitudes $\chi_{\alpha \beta}$ leads to the following eigenvalue problem

$$
\mathcal{S} \chi^{\mu}=\Omega_{\mu} \mathcal{N}_{1} \chi^{\mu}
$$

where

$$
\begin{aligned}
& \mathcal{S}\left(\alpha \alpha^{\prime}: \lambda \lambda^{\prime}\right)=\left\langle 0\left|\left[a_{\alpha^{\prime}}^{+} a_{\alpha},\left[H, a_{\lambda}^{+} a_{\lambda^{\prime}}\right]\right]\right| 0\right\rangle= \\
& \left(\epsilon_{\alpha}-\epsilon_{\alpha^{\prime}}\right)\left(n_{\alpha^{\prime}}-n_{\alpha}\right) \delta_{\alpha \lambda} \delta_{\alpha^{\prime} \lambda^{\prime}} \\
& +\left(n_{\alpha^{\prime}}-n_{\alpha}\right)\left(n_{\lambda^{\prime}}-n_{\lambda}\right) \bar{v}_{\alpha \lambda^{\prime} \alpha^{\prime} \lambda} \\
& -\delta_{\alpha^{\prime} \lambda^{\prime}} \frac{1}{2} \sum_{\gamma \gamma^{\prime} \gamma^{\prime \prime}} \bar{v}_{\alpha \gamma \gamma^{\prime} \gamma^{\prime \prime}} C_{\gamma^{\prime} \gamma^{\prime \prime} \lambda \gamma} \\
& -\delta_{\alpha \lambda} \frac{1}{2} \sum_{\gamma \gamma^{\prime} \gamma^{\prime \prime}} \bar{v}_{\gamma \gamma^{\prime} \alpha^{\prime} \gamma^{\prime \prime}} C_{\lambda^{\prime} \gamma^{\prime \prime} \gamma \gamma^{\prime}} \\
& +\sum_{\gamma \gamma^{\prime}}\left(\bar{v}_{\alpha \gamma \lambda \gamma^{\prime}} C_{\lambda^{\prime} \gamma^{\prime} \alpha^{\prime} \gamma}+\bar{v}_{\lambda^{\prime} \gamma \alpha^{\prime} \gamma^{\prime}} C_{\alpha \gamma^{\prime} \lambda \gamma}\right) \\
& -\frac{1}{2} \sum_{\gamma \gamma^{\prime}}\left(\bar{v}_{\alpha \lambda^{\prime} \gamma \gamma^{\prime}} C_{\gamma \gamma^{\prime} \alpha^{\prime} \lambda}+\bar{v}_{\gamma \gamma^{\prime} \alpha^{\prime} \lambda} C_{\alpha \lambda^{\prime} \gamma \gamma^{\prime}}\right), \\
& \mathcal{N}_{1}\left(\alpha \alpha^{\prime}: \lambda \lambda^{\prime}\right)=\left(n_{\alpha^{\prime}}-n_{\alpha}\right) \delta_{\alpha \lambda} \delta_{\alpha^{\prime} \lambda^{\prime}} .
\end{aligned}
$$

This is the same equation as the one-body part of (24), (25), (28). If we replace the RPA ground state by the HF one, then the matrix $\mathcal{S}$ reduces to the HF stability matrix and $\mathcal{N}_{1}^{(0)}$ becomes the metric matrix of RPA [12] and, thus, the standard RPA equations are recovered. The normalisation of the amplitudes $\chi_{\alpha \beta}^{\nu}$ is given by

$$
\sum \chi_{\alpha \beta}^{\nu *} \mathcal{N}_{1}\left(\alpha \alpha^{\prime}: \lambda \lambda^{\prime}\right) \chi_{\lambda \lambda^{\prime}}^{\nu^{\prime}}=\delta_{\nu, \nu^{\prime}},
$$

where $\chi_{\alpha \beta}^{\nu *}$ is the left eigenvector. The above eigenvalue problem is equivalent to SCRPA [16] with amplitudes $\chi_{\alpha \beta}$ where there are no restrictions on the indices besides $\alpha \neq \beta$. This stems from the fact that $\mathcal{N}_{1}$ acts as a norm matrix like it appears in problems where one works with a non-orthonormal basis [12]. In such cases, in general, one has to diagonalise the norm matrix and divide the Hamilton matrix from left and right with the square roots of the eigenvalues. Configurations with zero (or near-zero) eigenvalues have to be excluded for obvious reasons. In the SCRPA case, this just happens for diagonal, or nearly diagonal amplitudes $\chi_{\alpha \alpha}$ which, thus, cannot be included. This can only be done, as we discussed before, if the twoparticle sector is also considered.
The fact that the diagonal amplitudes cannot be included in (41) allows us to rewrite this equation in a form which has the mathematical structure of standard RPA. To this end, we rewrite the RPA excitation operator (37) in a somewhat different form, see also [25],

$$
Q_{\nu}^{+}=\sum_{k_{1}>k_{2}}\left(X_{k_{1} k_{2}}^{\nu} \delta Q_{k_{1} k_{2}}^{+}-Y_{k_{1} k_{2}}^{\nu} \delta Q_{k_{1} k_{2}}\right)
$$

with

$$
\delta Q_{k_{1} k_{2}}^{+}=N_{k_{1} k_{2}}^{-1 / 2} a_{k_{1}}^{+} a_{k_{2}}
$$

and

$$
N_{k_{1} k_{2}}^{1 / 2}=\sqrt{n_{k_{2}}-n_{k_{1}}} .
$$

This leads straightforwardly to the following RPA eigenvalue problem:

$$
\left(\begin{array}{cc}
A & B \\
-B^{*} & -A^{*}
\end{array}\right)\left(\begin{array}{l}
X \\
Y
\end{array}\right)=\Omega_{\nu}\left(\begin{array}{l}
X \\
Y
\end{array}\right)
$$

with

$$
\begin{aligned}
& A_{k_{1} k_{2}, k_{1}^{\prime} k_{2}^{\prime}}=\left\langle\left[\delta Q_{k_{1} k_{2}},\left[H, \delta Q_{k_{1}^{\prime} k_{2}^{\prime}}^{+}\right]\right]\right\rangle \\
& B_{k_{1} k_{2}, k_{1}^{\prime} k_{2}^{\prime}}=-\left\langle\left[\delta Q_{k_{1} k_{2}},\left[H, \delta Q_{k_{1}^{\prime} k_{2}^{\prime}}\right]\right]\right\rangle .
\end{aligned}
$$

The $X, Y$ amplitudes have the usual orthonormalisation relations of standard $p h$-RPA with the replacements $p \leftrightarrow k_{1}$ and $h \leftrightarrow k_{2}$. Of course the $A$ and $B$ matrices are closely related to the $\mathcal{S}$ matrix of (42).

In order to calculate the $C_{2}$ correlation functions entering the SCRPA matrix, one can either get them from the static solution of the TDDM equations with quadratic decoupling of $C_{3}$ with respect to the $C_{2}$ 's (this will later be called the C-RPA scheme) or one establishes a selfconsistent cycle, for which we must give a relation between $C_{2}$ and the RPA amplitudes $X, Y$. For this, it is convenient to introduce the "bosonic" density matrix $\mathcal{R}$

$$
\mathcal{R}=\sum_{\nu}\left(\begin{array}{ll}
Y^{\nu *} Y^{\nu} & Y^{\nu *} X^{\nu} \\
X^{\nu *} Y^{\nu} & X^{\nu *} X^{\nu}
\end{array}\right) \equiv\left(\begin{array}{cc}
R & K \\
K^{+} & 1+R^{+}
\end{array}\right),
$$

obeying the relation $\left(\mathcal{N}_{0} \mathcal{R}\right)^{2}=-\mathcal{N}_{0} \mathcal{R}$ where $\mathcal{N}_{0}=\left(\begin{array}{cc}1 & 0 \\ 0 & -1\end{array}\right)$ and where we can make the following identifications:

$$
\begin{aligned}
& R_{k_{1} k_{2} k_{1}^{\prime} k_{2}^{\prime}} \equiv N_{k_{1} k_{2}}^{-1 / 2}\left[n_{k_{2}} \bar{n}_{k_{1}}+C_{k_{1} k_{2}^{\prime} k_{2} k_{1}^{\prime}}\right] N_{k_{1}^{\prime} k_{2}^{\prime}}^{-1 / 2} \\
& K_{k_{1} k_{2} k_{1}^{\prime} k_{2}^{\prime}} \equiv N_{k_{1} k_{2}}^{-1 / 2} C_{k_{1} k_{1}^{\prime} k_{2} k_{2}^{\prime}} N_{k_{1}^{\prime} k_{2}^{\prime}}^{-1 / 2} .
\end{aligned}
$$

It may be interesting to rewrite the RPA equations in still a different form. With

$$
\left(\begin{array}{cc}
A & B \\
-B^{*} & -A^{*}
\end{array}\right) \equiv \mathcal{H}
$$

we can write (48) as

$$
\mathcal{R H}^{+}-\mathcal{H R}=0
$$

This form recalls the BCS (or HFB) equations of superconductivity [12] with, however, some different signs 


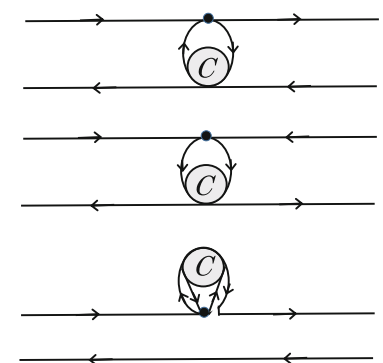

Fig. 2. Screening terms and self-energy corrections contained in the SCRPA kernel. Symmetric graphs exist where the interaction (full dot) is attached to the hole line (arrow to the left).

due to the bosonic structure of the RPA equations. The introduction of the density matrix $\mathcal{R}$ has the advantage that one can easily restore a missing antisymmetry.

It remains to express the occupation numbers in terms of the RPA amplitudes to establish fully self-consistent RPA equations. Because of the Fermi surface the occupation numbers can be divided in hole and particle occupancies $n_{h}$ and $n_{p}$. How the latter are connected to $C_{2}$ 's and, thus, to the RPA amplitudes will be shown in sect. 5 .

At this point, it may be appropriate to interpret the different terms of the $A$ and $B$ matrices. The standard terms are, of course, trivial and have been discussed in text books [12]. The other terms are displayed graphically in fig. 2. Analogous graphs exist (not displayed) where the interaction (full dot) is attached to the hole line with arrow to the left. Their interpretation is clear. The first two terms constitute instantaneous $p h$ and $p p(h h)$ exchange terms with respect to the external $p$ and $h$ lines. They, therefore, screen (eventually anti screen) the bare interaction. Such screening terms have been discussed in the literature since very long. The iteration of the equations gives raise to so-called "bubble into bubble" terms [26]. The particularity of our formalism here is that those terms emerge from a general formalism and that they are instantaneous. They can, therefore, be incorporated into standard RPA programs. The third term in fig. 2 obviously corresponds to a self-energy correction due to RPA modes. Those correspond to the famous particle vibration corrections to the mean field. Again the particularity here is that this correction is instantaneous.

For the solution of the SCRPA equations, several routes are possible. The standard way is to express the correlation functions by the $X$ and $Y$ amplitudes as discussed just above. With the present formalism one can also evaluate the correlation functions $C_{2}$ 's from (53) and then insert them into the $A$ and $B$ matrices. Also the single-particle occupancies can be included in this way via eq. (66), see sect. 5, below. The results will depend on whether we take the non-antisymmetrised or the antisymmetrised form of $\mathcal{R}$. Only the non-antisymmetrised form will be equivalent to the standard way in expressing everything by the $X, Y$ amplitudes. A further possibility is to take the $C_{2}$ 's directly from the static limit of the TDDM equations quadratic in the $C_{2}$ 's. As mentioned, we call this the C-RPA (correlated RPA). We will see with the applications in sect. 6 that all these variants give quite close answers at least up to coupling strengths where the standard HF equations become unstable indicating that the system undergoes a phase transition.

\subsection{Properties of SCRPA}

Let us outline some properties of SCRPA. One of the most important ones, fulfilled by the standard RPA, is the socalled energy weighted sum rule,

$$
S_{1}=\sum_{\nu} \Omega_{\nu}|\langle\nu|F| 0\rangle|^{2}=\frac{1}{2}\langle 0|[F,[H, F]]| 0\rangle,
$$

where $F=\sum_{\alpha \beta} f_{\alpha \beta} a_{\alpha}^{+} a_{\beta}$ is supposed to be a Hermitian one-body (excitation) operator. Then for the right-hand side we can write

$$
\begin{aligned}
S_{1} & =\frac{1}{2} \sum_{\nu} \operatorname{Tr}\left[f^{+} \chi^{\nu} S \chi^{\nu,+} f\right] \\
& =\frac{1}{2} \operatorname{Tr}\left[f^{+} \sum_{\nu} \Omega_{\nu} \mathcal{N}_{1} \chi^{\nu,+} f\right] \\
& =\sum_{\nu} \Omega_{\nu}|\langle 0|F| \nu\rangle|^{2} .
\end{aligned}
$$

Therefore also SCRPA fulfills the $f$-sum rule. This has, e.g., been discussed in [20,27-29]. From the fulfillment of the sum rule, it also follows that the Goldstone theorem is satisfied. For example in nuclear physics the translational motion is always broken, if one works in a localised single-particle basis. Then the SCRPA separates the socalled spurious mode at zero energy, if the single-particle basis is chosen from the generalised mean-field equation $\left\langle 0\left|\left[H, Q_{\nu}^{+}\right]\right| 0\right\rangle=\left\langle 0\left|\left[H, a_{\alpha^{\prime}}^{+} a_{\alpha}\right]\right| 0\right\rangle=0$ which is the static limit of the first equation of motion (2), see also [29]. This has been demonstrated analytically in [20]. Actually the Goldstone mode is already obtained employing the HF basis as long as one works with the $m$-scheme where antisymmetrisation can be fully and explicitly respected [20]. The fulfillment of the sum rule and the Goldstone mode stems from the fact that the RPA operator (37) contains all types of indices, that is not only $p h$ but also $p p$ and $h h$ ones. The RPA operator (37) contains as a particular case, e.g., the total momentum operator $\hat{P}$ which commutes with the Hamiltonian. From (40) we then see that the zero mode appears. The fulfillment of the Goldstone theorem has already explicitly been demonstrated in [25]. However, in that work it was crucial to satisfy (2) in defining a generalised single-particle basis. This is due to the fact that in [25] collective generators for the RPA operator were used what did not allow to fully respect antisymmetrisation. Consequently SCRPA as defined in this section has some important properties in common with standard RPA. This is a very rewarding feature because generally it is not easy to set up a practical scheme, going beyond standard RPA, which obeys conservation laws, sum rules, and the Goldstone theorem. However SCRPA 
is an approximation to STDDM-b (or STDDM*-b) and, therefore, also fails in some respects. For example in the superfluid (superconducting) case, the symmetry operator is the particle number operator which in the quasiparticle basis has a diagonal (Hermitian) piece. This cannot be included into SCRPA because the norm matrix (43) has a zero eigenvalue. Thus, self-consistent quasi-particle RPA will not give the zero mode. However, in infinite matter this is not a serious problem, since one can approach the zero mode asymptotically covering, thus, almost all of the Bogoliubov-Anderson Goldstone mode. For finite discrete systems, the consideration of the STDDM approach is necessary, which allows to include diagonal elements as explained in sect. 3.3. It may, however, be possible to include the 2-body sector only in approximate, eventually perturbative form. This possibility remains to be investigated.

Another important property of standard RPA which is fulfilled by SCRPA is gauge invariance. Gauge invariance of standard RPA is nicely demonstrated by Feldman and Fulton [30]. The extra terms containing the two-body correlation functions in (42) cancel in the limit where the two open legs are put on the same spot in position space. Actually, gauge invariance of standard RPA as well as SCRPA can easily be verified from (49). If in these equations the operator $\delta Q_{\alpha \beta}$ is transformed into $r$-space and the diagonal element is taken, as demanded to show gauge invariance (see [30], eq. (3.69)), we immediately realise that this diagonal operator commutes with the remainder (also written in $r$-space), once the Hamiltonian $H$ is replaced by its interaction part $V$, that is, the Coulomb interaction. Therefore, gauge invariance is fulfilled. This argument is valid discarding spin but, as shown in [30], this does not invalidate the general proof. These considerations also entail that the so-called "velocity-length" equivalence in the dipole transition is preserved [30], see also [29].

\section{Occupation numbers from SCRPA and the coupled cluster wave function}

To be self-contained, in this section, we will re-derive SCRPA from a different perspective [31]. This will have an interesting connection with TDDM and will give some insight into which kind of ground state is implicitly used in STDDM* and/or STDDM.

Formally the SCRPA equations have been written down several times in the past $[13,16]$ and references therein. They can be qualified as some sort of HartreeFock-Bogoliubov (HFB) equations for fermionic $p h$ pairs and they most of the time have been presented as a non-linear eigenvalue problem to be solved by iteration. SCRPA theory has recently known important new developments concerning its theoretical foundation [31]. This stems from the fact that it was shown in that reference that the CCD coupled cluster wave function

$$
\begin{aligned}
|Z\rangle & =e^{\hat{Z}}|\mathrm{HF}\rangle \\
\text { with } \quad \hat{Z} & =\frac{1}{4} \sum_{p_{1} p_{2} h_{1} h_{2}} z_{p_{1} p_{2} h_{1} h_{2}} a_{p_{1}}^{+} a_{p_{2}}^{+} a_{h_{1}} a_{h_{2}}
\end{aligned}
$$

is the vacuum to the following generalised RPA operator:

$$
\begin{aligned}
\tilde{Q}_{\nu}^{+}= & \sum_{p h}\left[\tilde{X}_{p h}^{\nu} a_{p}^{+} a_{h}-\tilde{Y}_{p h}^{\nu} a_{h}^{+} a_{p}\right] \\
& +\frac{1}{2} \sum_{p h p_{1} p_{2}} \eta_{p h p_{1} p_{2}} a_{p_{1}}^{+} a_{p_{2}} a_{h}^{+} a_{p} \\
& -\frac{1}{2} \sum_{p h h_{1} h_{2}} \eta_{h_{1} h_{2} p h} a_{h_{1}}^{+} a_{h_{2}} a_{h}^{+} a_{p}
\end{aligned}
$$

That is, there exists the killing condition

$$
\tilde{Q}_{\nu}|Z\rangle=0
$$

with the following relations between the various amplitudes

$$
\begin{aligned}
\tilde{Y}_{p h}^{\nu} & =\sum_{p^{\prime} h^{\prime}} z_{p p^{\prime} h h^{\prime}} \tilde{X}_{p^{\prime} h^{\prime}}^{\nu} \\
z_{p p^{\prime} h h^{\prime}} & =\sum_{\nu} \tilde{Y}_{p h}^{\nu}\left(\tilde{X}^{-1}\right)_{p^{\prime} h^{\prime}}^{\nu} \\
\eta_{p_{1} p_{2} p h}^{\nu} & =\sum_{h_{1}} z_{p p_{2} h h_{1}} \tilde{X}_{p_{1} h_{1}}^{\nu} \\
\eta_{h_{1} h_{2} p h}^{\nu} & =\sum_{p_{1}} z_{p p_{1} h h_{2}} \tilde{X}_{p_{1} h_{1}}^{\nu} .
\end{aligned}
$$

The amplitudes $z_{p p^{\prime} h h^{\prime}}$ are antisymmetric in $p p^{\prime}$ and $h h^{\prime}$. With the above relations, the vacuum state is entirely expressed by the RPA amplitudes $\tilde{X}, \tilde{Y}$. We remark that this vacuum state is exactly the one of coupled cluster theory (CCT) truncated at the two-body level $[9,32]$. However, the use we will make of this vacuum is very different from CCT. Of course, for the moment, all remains formal because this generalized RPA operator contains, besides the standard one-body terms, also specific twobody terms which cannot be handled in a straightforward way. For instance, this non-linear transformation cannot be inverted in a simple manner. However, we find the mere existence of an exact killing operator of the coupled cluster ground state quite remarkable. One may develop approximate methods to cope with those extra two-body terms. A first simple approximation consists in replacing in (57) the occupation number operators in the $\eta$ terms by their expectation values, that is $a_{p_{2}}^{+} a_{p_{1}} \rightarrow\left\langle a_{p_{1}}^{+} a_{p_{1}}\right\rangle \delta_{p_{1} p_{2}}$ and $a_{h_{1}}^{+} a_{h_{2}} \rightarrow\left\langle a_{h_{1}}^{+} a_{h_{1}}\right\rangle \delta_{h_{1} h_{2}}$ where we supposed that we work in a basis where the single-particle density matrix is diagonal. With the definition of the occupation numbers $n_{k}=\left\langle a_{k}^{+} a_{k}\right\rangle$, we then obtain the following approximate form of the $Q$-operator in (57):

$$
\begin{aligned}
\tilde{Q}_{\nu}= & \sum_{p h}\left[\tilde{X}_{p h}^{\nu} a_{h}^{+} a_{p}-\tilde{Y}_{p h}^{\nu} a_{p}^{+} a_{h}\right] \\
& +\frac{1}{2} \sum_{p h p_{1}} \eta_{p_{1} p_{1} p h} n_{p_{1}} a_{p}^{+} a_{h} \\
& -\frac{1}{2} \sum_{p h h_{1}} \eta_{h_{1} h_{1} p h} n_{h_{1}} a_{p}^{+} a_{h} .
\end{aligned}
$$


Evidently, this approximation, though suggestive, violates the killing condition (58). However, as has been shown in [31], the violation remains quite moderate. On the other hand, this approximation leads to a renormalisation of the $\tilde{Y}$ amplitudes in (60) and, therefore, we are back to the usual RPA operator with the one-body terms in (60) only. For simplicity, we will not change the nomenclature of the $\tilde{Y}$ amplitudes in the following. In spite of the approximation, we will henceforth assume that the killing condition still holds. However, we always should be aware that this only is true approximately with the atrophied form of the generalized RPA operator (60). The amplitudes $(\tilde{X}, \tilde{Y})$ form a complete orthogonal set of vectors as explained, e.g., in [12]. We therefore can invert the approximate RPA operator to obtain

$$
a_{p}^{+} a_{h}=\sqrt{N_{p h}} \sum_{\nu}\left[X_{p h}^{\nu} Q_{\nu}^{+}+Y_{p h}^{\nu} Q_{\nu}\right]
$$

where we defined new amplitudes $X, Y$ via

$$
\tilde{X}_{p h}^{\nu}=X_{p h}^{\nu} / \sqrt{N_{p h}}, \quad \tilde{Y}_{p h}^{\nu}=Y_{p h}^{\nu} / \sqrt{N_{p h}}
$$

and new RPA operators

$$
Q_{\nu}=\sum_{p h}\left[X_{p h}^{\nu} a_{h}^{+} a_{p}-Y_{p h}^{\nu} a_{p}^{+} a_{h}\right] / \sqrt{N_{p h}}
$$

so that the state $|\nu\rangle=Q_{\nu}^{+}|Z\rangle$ is normalized, i.e., $\langle\nu \mid \nu\rangle=$ $\left\langle Z\left|\left[Q_{\nu}, Q_{\nu}^{+}\right]\right| Z\right\rangle /\langle Z \mid Z\rangle=1$ with

$$
\sum_{p h}\left[\left|X_{p h}^{\nu}\right|^{2}-\left|Y_{p h}^{\nu}\right|^{2}\right]=1
$$

The use of the CCT state $|Z\rangle$ has the great advantage that now in the calculation of the expectation values where we also need the occupation numbers expressed in terms of the $X, Y$ amplitudes, this can be achieved in a natural manner (this was in the past always a certain problem with SCRPA without the use of the CCT state). For example, we have

$$
a_{h}^{+} a_{h}|Z\rangle=e^{\hat{Z}} \tilde{J}_{h h}|\mathrm{HF}\rangle,
$$

with $J_{h h}=a_{h}^{+} a_{h}$ and $\tilde{J}_{h h}=e^{-\hat{Z}} J_{h h} e^{\hat{Z}}=J_{h h}+\left[J_{h h}, \hat{Z}\right]$. Evaluating the commutator and then using the relation

$$
\sum_{\nu}\left(\tilde{X}^{-1}\right)_{p^{\prime} h^{\prime}}^{\nu} Q_{\nu}=a_{h^{\prime}}^{+} a_{p^{\prime}}-\sum_{p h} z_{p p^{\prime} h h^{\prime}} a_{p}^{+} a_{h}
$$

we arrive at

$$
\begin{aligned}
n_{h} & =\left\langle a_{h}^{+} a_{h}\right\rangle \equiv \frac{\left\langle Z\left|a_{h}^{+} a_{h}\right| Z\right\rangle}{\langle Z \mid Z\rangle} \\
& =1-\frac{1}{2} \sum_{p}\left\langle a_{p}^{+} a_{h} a_{h}^{+} a_{p}\right\rangle \\
& =1-\frac{1}{2} \sum_{p}\left[n_{p} \bar{n}_{h}-C_{p h p h}\right] .
\end{aligned}
$$

This relation can be used in (53) to have a fully closed system of equations. For the evaluation of the two-body term in terms of the $Y$-amplitudes, we will use the inversion of the $Q$-operators and obtain

$$
n_{h} \equiv\left\langle a_{h}^{+} a_{h}\right\rangle=1-\frac{1}{2} \sum_{p, \nu}\left(n_{h}-n_{p}\right)\left|Y_{p h}^{\nu}\right|^{2} .
$$

The same can be repeated for $n_{p}$,

$$
\begin{aligned}
n_{p} \equiv\left\langle a_{p}^{+} a_{p}\right\rangle & =\sum_{h} \frac{1}{2} \sum_{h}\left\langle a_{p}^{+} a_{h} a_{h}^{+} a_{p}\right\rangle \\
& =\frac{1}{2} \sum_{h}\left[n_{p} \bar{n}_{h}-C_{p h p h}\right] \\
& =\frac{1}{2} \sum_{h, \nu}\left(n_{p}-n_{h}\right)\left|Y_{p h}^{\nu}\right|^{2},
\end{aligned}
$$

leading to a linear system of equations for $n_{p}, n_{h}$ which can be solved. The quadratic occupation number fluctuations can be treated in a similar way. They are related to $C_{2}$ 's with either four particle or four hole indices. They can be approximated to leading order by quadratic forms of $C_{2}$ 's with $p p h h$ indices as shown in [10],

$$
\begin{aligned}
C_{p_{1} p_{2} p_{3} p_{4}} & \simeq \frac{1}{2} \sum_{h h^{\prime}} C_{p_{1} p_{2} h h^{\prime}} C_{h h^{\prime} p_{3} p_{4}}+\ldots \\
C_{h_{1} h_{2} h_{3} h_{4}} & \simeq \frac{1}{2} \sum_{p p^{\prime}} C_{h_{1} h_{2} p p^{\prime}} C_{p p^{\prime} h_{3} h_{4}}+\ldots
\end{aligned}
$$

We now can express all correlation functions and densities in $A$ and $B$ matrices by the RPA amplitudes $X, Y$ and, thus, have a fully self-consistent system of equations for $X, Y$. It should be mentioned, however, that due to the fact that the present RPA operator only contains $p h(h p)$ configurations, sum rules, Goldstone theorem, etc. are not strictly fulfilled. The violations usually remain very weak though, see [31].

There exists, however, a different closing of the equations employing the so-called self-consistent particleparticle RPA (SCppRPA $[33,34])$. It can be shown that the coupled cluster wave function is not only the vacuum to a generalised RPA operator in the $p h$ channel but also in the $p p(h h)$ channel. This is explained in ref. [31]. From SCppRPA one can naturally obtain the $C_{2}$ 's with four particle or four hole indices, that is $C_{p_{1} p_{2} p_{3} p_{4}}$ and $C_{h_{1} h_{2} h_{3} h_{4}}$. Also the SCppRPA couples via the non-linearity back to the particle-hole SCRPA considered here [16].

Iterating SCphRPA and SCppRPA simultaneously sums up $p h$ and $p p(h h)$ correlations in a democratic way.

\section{Applications to Lipkin and Hubbard models}

\subsection{Preliminaries}

In order to guide the reader in the following applications with the various approximations used, let us make a short summary here.

First, there is the TDDM method, described in sect. 2 . It allows to calculate the occupation numbers $n_{k}$ and the 
four types of 2-body correlation functions considered. Disposing of those quantities allows to calculate the total ground-state energy or various partial quantities thereof, as, e.g., the so-called 2-body correlation energy. The $n_{k}$ and $C_{2}$ 's so obtained can also be used to set up the correlated RPA matrix, in which case we talk about the CRPA scheme. The C-RPA and SCRPA schemes appear naturally as the one-body sector of the linearised TDDM equations. The latter equations have been called either STDDM*-b or STDDM-b equations according to whether one includes the approximate form of the 3-body correlation function $C_{3}$, eq. (12), or not. Let us recall that the structure of the one-body sector of STDDM-b and STDDM*-b is not affected by $C_{3}$ when the 2-body space is decoupled from the 1-body one. The non-linearity in $\mathrm{C}_{2}$ 's only affects the 2-body sector as seen when comparing (25) with (28). There also exist STDDM and STDDM* equations which are equivalent but very non-symmetric versions of STDDM-b and STDDM*-b, respectively. Finally there exists the so-called Extended Second RPA (ESRPA) equation which does not follow from the TDDM approach but is obtained from a minimisation of the energy weighted sum rule involving 1-body and 2-body operators. Since STDDM-b and STDDM*-b equations can be shown to be approximate forms of ESRPA, we consider, in principle, ESRPA somewhat superior to all the other kinds of equations we have established. One should realise, however, that STDDM-b, STDDM*-b, and ESRPA which all include the 2-body sector can be solved for the model cases presented below which involve limited configuration spaces but for realistic problems, such as the homogeneous electron gas or nuclear matter, etc., one must be content if the equations of the 1-body sector, that is C-RPA and/or SCRPA can be tackled with the presentday computer powers. This, for instance, in the light that, e.g., STDDM equations have to be solved in full space to preserve their properties concerning conservation laws and Goldstone theorem (see also discussion at the end of this paper). One should appreciate the following results in the light of these preliminary remarks.

\subsection{Lipkin model}

We first consider the Lipkin model [35]. The Lipkin model describes an $N$-fermions system with two $N$-fold degenerate levels with energies $\epsilon / 2$ and $-\epsilon / 2$, respectively. The upper and lower levels are labeled by quantum number $1 \mathrm{~m}$ and $0 m$, respectively, with $m=1,2, \ldots, N$. We consider the standard Hamiltonian

$$
\hat{H}=\epsilon \hat{J}_{z}+\frac{V}{2}\left(\hat{J}_{+}^{2}+\hat{J}_{-}^{2}\right)
$$

where the operators are given as

$$
\begin{aligned}
& \hat{J}_{z}=\frac{1}{2} \sum_{m=1}^{N}\left(a_{1 m}^{+} a_{1 m}-a_{0 m}{ }^{+} a_{0 m}\right), \\
& \hat{J}_{+}=\hat{J}_{-}^{+}=\sum_{m=1}^{N} a_{1 m}^{+} a_{0 m} .
\end{aligned}
$$

The operators $J_{z}, J_{ \pm}$are pseudospin operators and fulfill commutation relations of angular momenta.

The ground state in TDDM is obtained using the adiabatic method: Starting from the HF ground state, we solve the TDDM equations (eqs. (2) and (3)) by gradually increasing the residual interaction such that $V^{\prime}=V \times t / T$, as described in [14]. We use $T=4 \times 2 \pi / \epsilon$. For the 3 body terms in eq. (3) we use the approximations eqs. (16) and (17) which are supposed to be the leading terms. All possible single-particle indices are taken into account one by one (the so-called $m$-scheme, see also [36]). The original basis is kept.

In a first application, the occupation numbers $n_{\alpha}$ and 2-body correlation functions $C_{\alpha \beta \alpha^{\prime} \beta^{\prime}}$ are determined from the TDDM calculation and the RPA matrix is set up with these values. We refer to this scheme as the correlated RPA (C-RPA), see sect. 4.1, to distinguish it from SCRPA which takes into account self-consistency. We found that the factor $1 / 2$ in eq. (67) is essential when we consider non-collective amplitudes as $\chi_{0 m^{\prime}, 1 m}^{\mu}$ and $\chi_{1 m, 0 m^{\prime}}^{\mu}$ in addition to $\chi_{0 m, 1 m}^{\mu}$ and $\chi_{1 m, 0 m}^{\mu}$, that is all possible RPAamplitudes. This is in line with the straightforward derivation which leads to the expression (67). When we keep only the collective amplitudes, the results deteriorate and in addition the factor $1 / 2(67)$ has to be suppressed. This is in agreement with the discussion about the factor $1 / 2$ in the occupation number expressions by Rowe in $[21,22]$ given a long time ago. Let us, however, stress again that in our formalism we should work in the full space.

In a second application we also performed selfconsistent RPA calculations corresponding to eq. (41), taking again all kinds of amplitudes, collective and noncollective, that is, we also included all the amplitudes $\chi_{0 m^{\prime}, 1 m}^{\mu}$ and $\chi_{1 m, 0 m^{\prime}}^{\mu}$ and consequently the factor $1 / 2$ in eq. (67) was kept. In SCRPA the two-body correlation matrices $C_{p_{1} p_{2} p_{3} p_{4}}$ and $C_{h_{1} h_{2} h_{3} h_{4}}$ which are not directly related to the one-body transition amplitudes $(X, Y)$ are calculated using eqs. (69) and (70). To calculate the $2 p$ - $2 h$ elements figuring in the above expressions for $C_{1 m 1 m^{\prime} 0 m 0 m^{\prime}}$ of the two-body correlation matrix, we use their relation with the RPA amplitudes given in eq. (51) with eq. (50). The occupation probability $n_{1 m}$ of the upper state and the two-body correlation matrix $C_{1 m 1 m^{\prime} 0 m 0 m^{\prime}}$ calculated in TDDM (solid line) and ESRPA (red squares) are shown in figs. 3 and 4, respectively, as a function of $\chi=(N-1)|V| / \epsilon$ for $N=4$. The RPA solution becomes unstable at $\chi=1$ as shown below in fig. 6 . The results of SCRPA (round dots) are shown up to $\chi=1.6$ because beyond $\chi \approx 1.6$ the numerical solution becomes unstable.

The Lipkin model is simple enough to solve the complicated self-consistent ESRPA equations (24), however still some approximations have been applied. For the three-body correlation functions, again the approximations eqs. (16) and (17) are employed. The 4-body correlation functions $C_{4}$ contained in the $\mathcal{D}$-matrix are neglected. Furthermore, in the ESRPA calculations we included only the one-body amplitudes with the same quantum number (this corresponds to the collective subspace as usually 


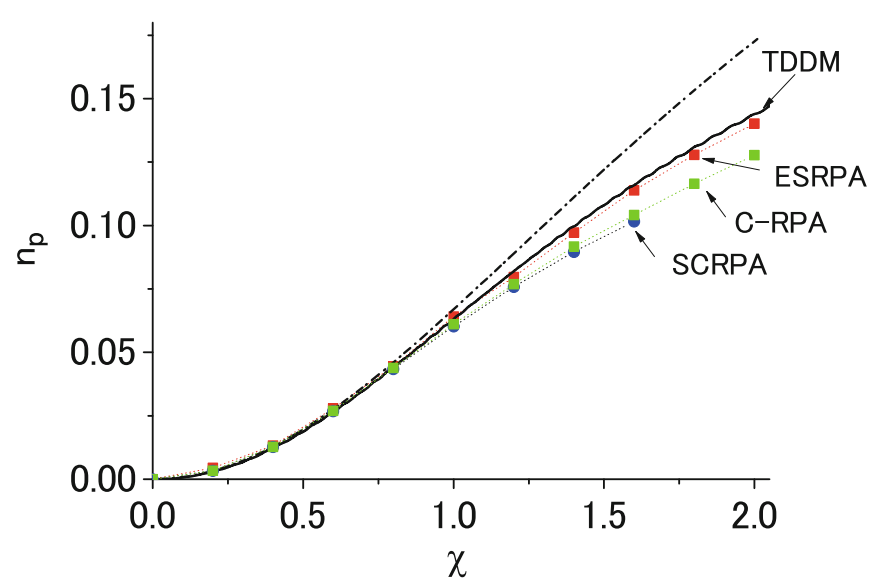

Fig. 3. Occupation probabilities $n_{p} \equiv n_{1 m}$ of the upper state calculated in TDDM (solid line), ESRPA (red squares), CRPA (green squares) and SCRPA (blue circles) as a function of $\chi=(N-1)|V| / \epsilon$ for $N=4$. The exact solution is shown with the dot-dashed line. The occupation probability and correlation matrix in TDDM are used in the C-RPA and ESRPA calculations.

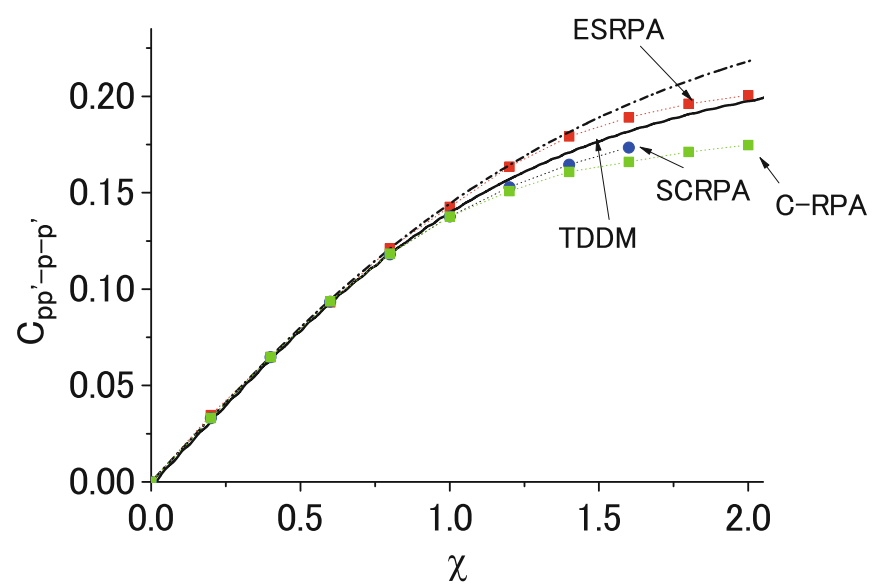

Fig. 4. Same as fig. 3 but for the two-body correlation matrix $C_{p p^{\prime}-p-p^{\prime}} \equiv C_{1 m 1 m^{\prime} 0 m 0 m^{\prime}}$.

considered in RPA) such as $\chi_{0 m, 1 m}^{\mu}, \chi_{1 m, 0 m}^{\mu}, \chi_{0 m, 0 m}^{\mu}$ and $\chi_{1 m, 1 m}^{\mu}$ and used eq. (31) to obtain $n_{1 m}$.

All two-body amplitudes $\mathcal{X}_{\alpha \beta \alpha^{\prime} \beta^{\prime}}$ with either $p_{\alpha}=p_{\alpha^{\prime}}$; $p_{\beta}=p_{\beta^{\prime}}$ or $p_{\alpha}=p_{\beta^{\prime}} ; p_{\beta}=p_{\alpha^{\prime}}$ are included, where $p_{\alpha}$ is the $1 m$ quantum number given in eqs. (72) and (73).

It should be mentioned that, since in the Lipkin model the even or odd number of $p h$ excitations decouple, the ESRPA equations also decouple into two subsets for even and odd parity. For example in fig. 6, the second excited state corresponds to even parity whereas the first one is of odd parity. Equally for the occupation numbers in fig. 3 only the odd parity amplitudes enter. Furthermore, since the ESRPA matrix has been set up with the results from TDDM, concerning $n_{k}$ and $C_{2}$ 's, one appreciates the numerical consistency when at the end the occupation numbers and two-body correlation functions are calculated from the ESRPA amplitudes as done in fig. 3 and fig. 4.

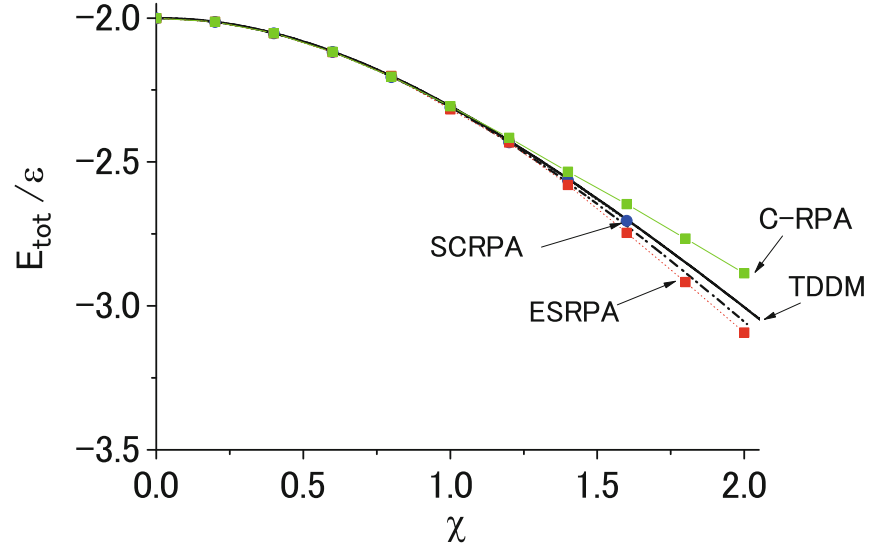

Fig. 5. Same as fig. 3 but for the ground-state energy.

The occupation numbers and 2-body correlation functions shown in figs. 3 and 4 are very sensitive quantities concerning the underlying wave function. Let us mention again that it is important for the accuracy of the results to work with all possible amplitudes (collective and noncollective), that is with the $m$-scheme. Taking into account only collective amplitudes sensitively deteriorates the results (not shown in the figures). SCRPA and C-RPA are about on same grounds, since they both work with the $m$-scheme and take the non-linearities in the $C_{2}$ 's into account. ESRPA and TDDM are also more or less equivalent, since they both take into account two-body amplitudes, see sect. 3.3. We may, however, remark that in realistic situations ESRPA may be inapplicable, besides in very restricted configuration spaces, because of its numerical complexity whereas this is not the case with TDDM. As a general remark, we can say that all approximations perform quite well up to $\chi=1$ but start to deviate more or less strongly from the exact result (dot-dashed line) thereafter. SCRPA and C-RPA are simpler than the approaches including the two-body sector because the dimensions of the matrices remain much smaller in the first case. The value $\chi=1$ is the one where standard RPA becomes unstable and a change of the single-particle basis becomes necessary (the "deformed" basis). Here, we do not operate a change of basis but still the system seems to feel the entering into a new "phase". We should also remember that $N=4$, is the worst case, see [31] where the quantum fluctuations are the strongest (the $N=2$ case, being more or less trivial, becomes exact in SCRPA; this is also the case in the 1D Hubbard model, treated below, see [37]). The results improve for higher values of $N$.

The ground-state energies in TDDM (solid line), ESRPA (red squares), C-RPA (green squares) and SCRPA (blue circles) are shown in fig. 5 as a function of $\chi$ for $N=4$. The exact values are again given with the dotdashed line. The ground-state energy in ESRPA is calculated using $n_{1 m}$ and $C_{1 \mathrm{~m} 1 \mathrm{~m}^{\prime} 0 \mathrm{mom}}$ given in figs. 3 and 4 . All calculations agree well with the exact values. The ground-state energy is a more robust quantity than, e.g., the occupation numbers.

The excitation energies of the first and second excited states are displayed in fig. 6 as a function of $\chi$. We see 


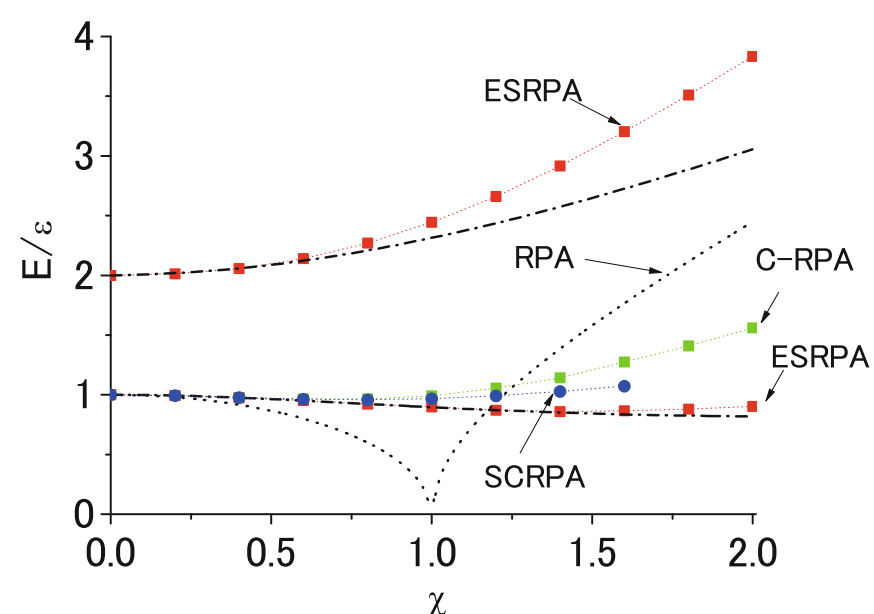

Fig. 6. Same as fig. 3 but for the excitation energies of the first and second excited states.

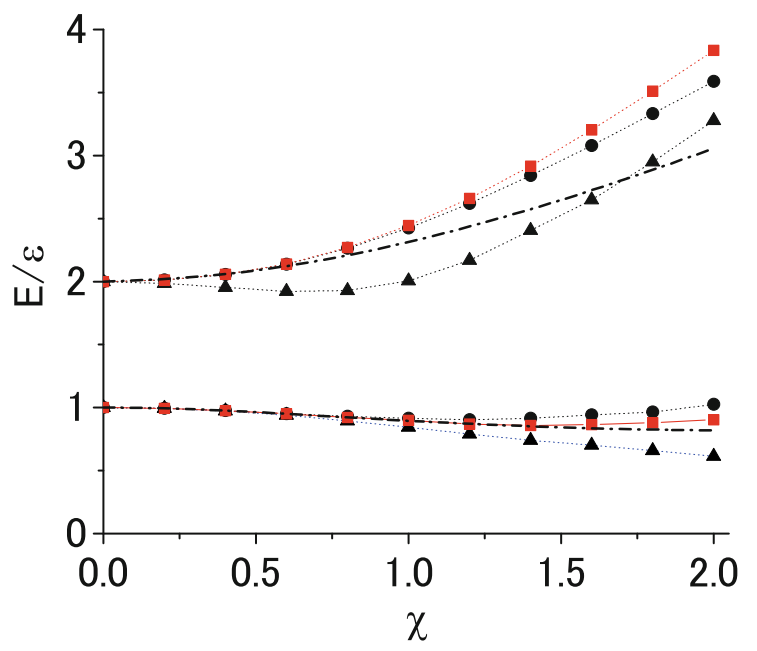

Fig. 7. Excitation energies of the first and second excited states calculated in STDDM-b (triangles), STDDM ${ }^{*}$-b (circles) and ESRPA (squares) as a function of $\chi=(N-1)|V| / \epsilon$ for $N=4$. The exact solution is shown with the dot-dashed line.

that ESRPA performs extremely well, even far beyond the RPA instability point of $\chi=1$. C-RPA and SCRPA also are very good but deteriorate after the instability point. Apparently the self-consistency (SCRPA) brings, in the domain where the results are stable, a slight advantage over the non-self-consistent one (C-RPA) but this may not be very significant in general cases. In the case of the second excited state which can be obtained with ESRPA, deviation from the exact solution becomes larger with increasing $\chi$. This can be explained either by the neglect of the coupling to higher amplitudes or by the fact that in ESRPA non-collective amplitudes are not included. The excitation energies of the first and second excited states calculated in STDDM-b (triangles), STDDM*-b (circles) and ESRPA (squares) are shown in fig. 7 as a function of $\chi=(N-1)|V| / \epsilon$ for $N=4$. The exact solution is again shown with the dot-dashed line. Figure 7 shows that STDDM*-b is a good approximation to ESRPA but up to $\chi=1$, STDDM-b also works quite well. All two- body amplitudes have been taken into account, that is $\mathcal{X}_{1 m 1 m^{\prime} 0 m 0 m^{\prime}}, \mathcal{X}_{0 m 0 m^{\prime} 1 m 1 m^{\prime}}, \mathcal{X}_{1 m 0 m^{\prime} 1 m 0 m^{\prime}}, \mathcal{X}_{1 m 1 m^{\prime} 1 m 1 m^{\prime}}$, $\mathcal{X}_{0 m 0 m^{\prime} 0 m 0 m^{\prime}}, \mathcal{X}_{1 m 0 m^{\prime} 0 m 0 m^{\prime}}, \mathcal{X}_{0 m 0 m^{\prime} 0 m 1 m^{\prime}}, \mathcal{X}_{1 m 1 m^{\prime} 1 m 0 m^{\prime}}$, and $\mathcal{X}_{0 \mathrm{~m} 1 \mathrm{~m}^{\prime} 1 \mathrm{~m} 1 \mathrm{~m}^{\prime}}$ where the last four terms are of odd parity and the first five terms of even parity.

Let us remind that the difference between ESRPA and STDDM-b and STDDM*-b is that in STDDM $C_{3}$ is totally neglected. The small difference between STDDM*-b and ESRPA originates in the fact that the matrix $\mathcal{D}$ in eq. (24) is not the same as $c \mathcal{T}+\tilde{d} \mathcal{N}_{2}$ in eq. (28). Otherwise the remarks as given above for the ESRPA results prevail also for STDDM-b and STDDM*-b.

\subsection{Hubbard model}

Finally we consider the one-dimensional (1D) Hubbard model with periodic boundary conditions. In the momentum space the Hamiltonian is given by

$$
\begin{aligned}
H= & \sum_{\boldsymbol{k}, \sigma} \epsilon_{k} a_{\boldsymbol{k}, \sigma}^{+} a_{\boldsymbol{k}, \sigma} \\
& +\frac{U}{2 N} \sum_{\boldsymbol{k}, \boldsymbol{p}, \boldsymbol{q}, \sigma} a_{\boldsymbol{k}, \sigma}^{+} a_{\boldsymbol{k}+\boldsymbol{q}, \sigma} a_{\boldsymbol{p},-\sigma}^{+} a_{\boldsymbol{p}-\boldsymbol{q},-\sigma}
\end{aligned}
$$

where $U$ is the on-site Coulomb matrix element, $\sigma$ is the spin projection and the single-particle energies are given by $\epsilon_{k}=-2 t \sum_{d=1}^{D} \cos \left(k_{d}\right)$ with the nearest-neighbor hopping potential $t$. We consider the case of six sites at half filling. In the first Brillouin zone $-\pi \leq k<\pi$ there are the following wave numbers:

$$
\begin{aligned}
& k_{1}=0, \quad k_{2}=\frac{\pi}{3}, \quad k_{3}=-\frac{\pi}{3}, \\
& k_{4}=\frac{2 \pi}{3}, \quad k_{5}=-\frac{2 \pi}{3}, \quad k_{6}=-\pi .
\end{aligned}
$$

The single-particle energies are $\epsilon_{1}=-2 t, \epsilon_{2}=\epsilon_{3}=-t$, $\epsilon_{4}=\epsilon_{5}=t$ and $\epsilon_{6}=2 t$. The ground state in TDDM is obtained using the adiabatic method starting from the HF ground state where the six lowest-energy single-particle states are completely occupied: $T$ used here is $5 \times 2 \pi / t$. The mean-field energy in ESRPA is calculated from the occupation probabilities given by eq. (31) and the correlation energy in ESRPA given by eq. (36): The first term on the right-hand side of eq. (35) vanishes due to $p$ - $h$ symmetry in the case of half-filling considered here. In SCRPA all $p$ - $h$ and $h-p$ amplitudes are taken and the factor $1 / 2$ in eq. (67) is kept. That is, we considered the following RPA excitation operator:

$$
Q_{\nu}^{+}=\sum_{p h}\left[X_{p h}^{\nu} a_{p}^{+} a_{h}-Y_{p h}^{\nu} a_{h}^{+} a_{p}\right],
$$

where $p, h=(\mathbf{p}, \mathbf{h}, \sigma)$ includes momenta and spin indices. Of course, in the end the SCRPA matrix will turn out to be block-diagonal in transferred momenta and charge and spin quantum numbers. However, in the set up of the SCRPA matrix, in the construction of the two-body 


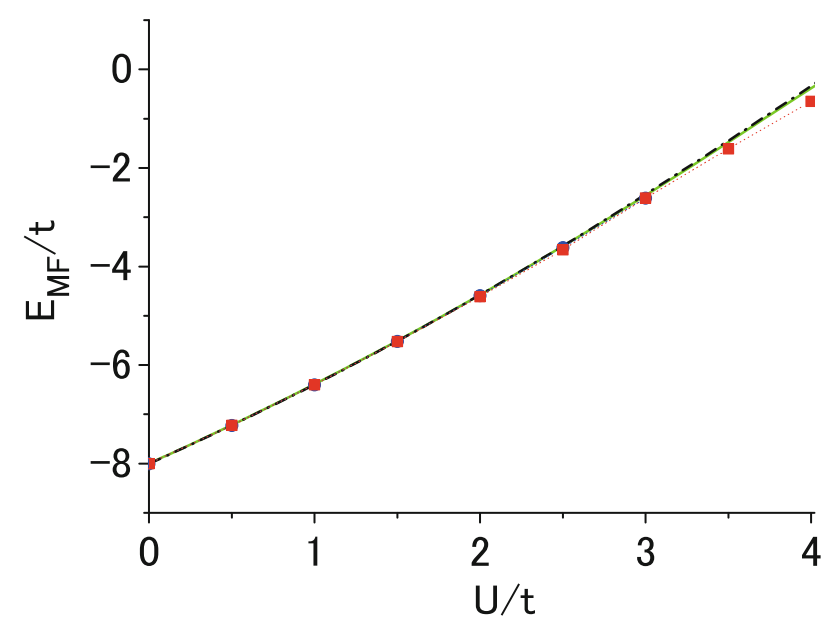

Fig. 8. Mean-field energy $E_{\mathrm{MF}}$ calculated in ESRPA (squares) and SCRPA (circles) as a function of $U / t$ for the six-site Hubbard model with half-filling. The TDDM results and the exact values are shown with the solid and dot-dashed lines, respectively.

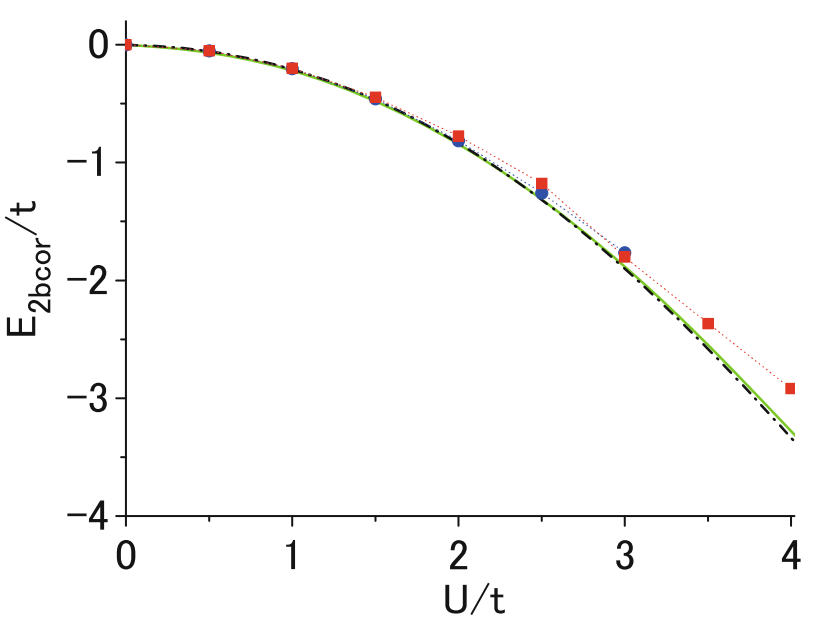

Fig. 9. Same as fig. 8 but for the 2-body correlation energy $E_{2 \text { bcor. }}$.

correlation functions all possible contributions are kept. Therefore, there is indirect coupling between all channels. This is different from [37] where the channels have been decoupled.

The matrix elements $C_{p_{1} p_{2} p_{3} p_{4}}$ and $C_{h_{1} h_{2} h_{3} h_{4}}$ are calculated using eqs. (69) and (70) in SCRPA. In the ESRPA calculations we take only the $2 p-2 h$ and $2 h-2 p$ components of $\mathcal{X}_{\alpha \beta \alpha^{\prime} \beta^{\prime}}^{\mu}$ to facilitate the numerics. Since the three-body correlation matrix is an approximate one, the stationary condition for the three-body correlation matrix is not completely fulfilled, which makes the Hamiltonian matrix of eq. (24) non-Hermitian, especially in the case of the Hubbard model which has more general two-body interaction than the Lipkin model.

The mean-field energy

$$
E_{\mathrm{MF}}=\sum_{\mathbf{k}}, \sigma \epsilon_{\mathbf{k}} n_{\mathbf{k}, \sigma}+\frac{U}{2 N} \sum_{\mathbf{k}, \mathbf{p}, \sigma} n_{\mathbf{k}, \sigma} n_{\mathbf{p},-\sigma},
$$

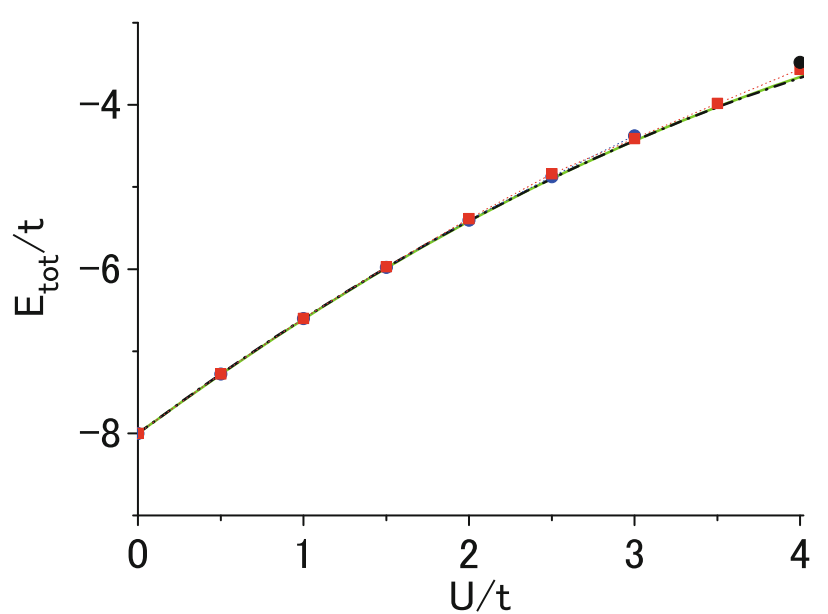

Fig. 10. Same as fig. 8 but for the ground-state energy $E_{\text {tot }}$.

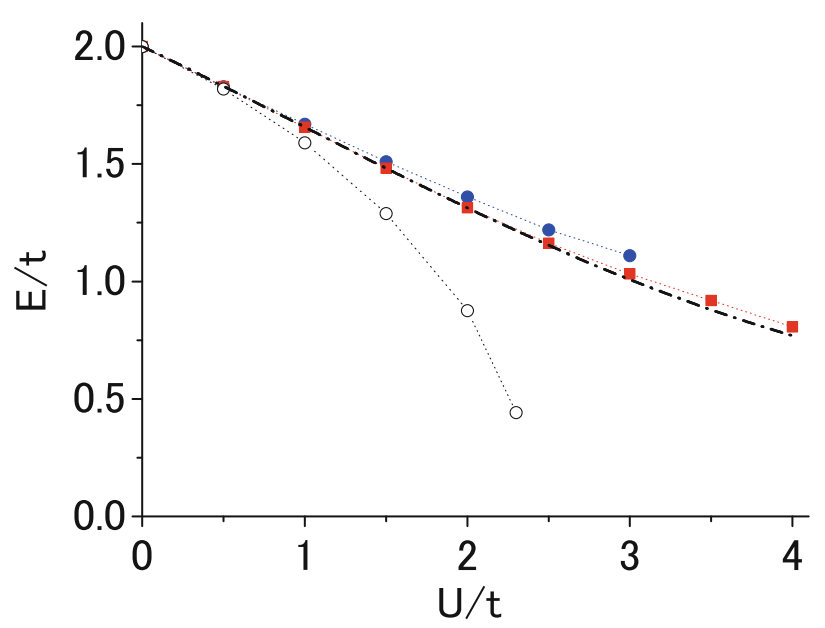

Fig. 11. Excitation energy of the first excited state calculated in SCRPA (circles) and ESRPA (squares) as a function of $U / t$ for the six-site Hubbard model with half-filling. The exact values are shown with the dot-dashed line. The open circles depict the results in RPA.

the correlation energy $E_{2 \mathrm{bcor}}$ and the ground-state energy $E_{\text {tot }}$ calculated in ESRPA (squares) are shown in figs. $8-10$ as a function of $U / t$. The results in SCRPA are also given with the circles up to $U / t=3$ but cannot be distinguished from those in ESRPA. Beyond $U / t \approx 3$, SCRPA cannot give meaningful solutions because of numerical instabilities as is the case for the Lipkin model. The TDDM results and the exact values are shown with the solid and dot-dashed lines, respectively. The results in ESRPA agree well with those in TDDM. The excitation energy of the first excited state is shown in fig. 11 as a function of $U / t$. The results in ESRPA (squares) show good agreement with the exact values (dot-dashed line). The SCRPA results (blue circles) are reasonable and avoid the instability of RPA (open circles). The SCRPA results in fig. 11 are, however, less good than the ones in [37]. For the second excited state (not shown) the situation becomes even worse. This fact needs some discussion. The reason for the present SCRPA results for the excitation 
energies apparently is due to the implicit cross channel couplings meaning that in the block matrix belonging to, e.g., a certain momentum transfer $q$, implicitly via the non-linear terms other momentum transfers also can enter. In [37], we discarded those "intruder" channels for the following reason: since SCRPA does not strictly satisfy the killing condition (58), the various RPA operators $Q_{\nu}^{+}$are not independent of one another. In [31], it was shown that this violation of independence is very weak. Apparently it is, however, still strong enough to perturb the equilibrium of the screening terms in the case of excitation energies. For correlation functions and ground-state energies, the problem seems to be much less severe. It may, thus, be better to discard the implicit channel coupling for the excitation energies in SCRPA which is an approximation to ESRPA or STDDM* (STDDM*-b). The channel couplings can be restored if the two-body amplitudes are taken care of as is seen in fig. 11 which, however, renders the problem much harder to be solved.

\subsection{Summary of applications}

We applied TDDM, STDDM-b and STDDM*-b, ESRPA, SCRPA, and C-RPA to two exactly solvable models. We found that the ground-state properties obtained from the excites states in ESRPA agree well with those in TDDM and the exact values. This indicates that the TDDM equations (eqs. (2) and (3)) build a ground state which is consistent with excited states. We also found that the results of SCRPA (and C-RPA) agree with those in ESRPA except for strongly interacting regions where the systems enter a new phase as, e.g., "deformation" in the case of the Lipkin model or anti-ferromagnetism in the case of the Hubbard model.

Let us mention that the TDDM scheme has also been tested in an eventually more realistic problem, see [38].

\section{Conclusions and discussions}

In this contribution, we outlined a new decoupling scheme of the Time-Dependent Density Matrix (TDDM) approach. Instead of neglecting totally the genuine threebody correlations, we approximated them by keeping an important sub-class of diagrams. Since the three-body correlations are of the $2 p-1 h$ or $2 h-1 p$ type, there exist contributions which are one fermion line reducible. The vertices for $2 p-1 h(2 h-1 p)$ to $1 p(1 h)$ transitions involve twobody correlation functions. Since those transitions intervene twice, one has a quadratic form in $C_{2}$ 's for the threebody correlations and, thus, the system of equations is closed on the level of $C_{2}$ 's. In applications to exactly solvable models, it turned out that the inclusion of this approximate form of three-body correlations is very important. We further linearised the new equations around equilibrium. This gives rise to extended second RPA equations, coupling the one-body sector to the two-body one. We named those equations the STDDM*-b equations. The one-body sector turns out to have the structure of the
Self-Consistent RPA (SCRPA) which was derived independently earlier. SCRPA, contrary to standard RPA, is built on a correlated ground state. Since this correlated ground state contains RPA correlations, naturally, one ends up with a self-consistency problem. Of course, neglecting correlations in the ground state, the STDDM*-b equations reduce to the standard second RPA ones. As an important side product, we could show that SCRPA as well as STDDM*-b conserve all the appreciated properties of standard RPA, i.e., fulfillment of sum rules, conservation laws, appearence of Goldstone (zero) modes in cases of spontaneously broken symmetries, and gauge invariance in the case of charged systems.

We applied our approaches to two exactly solvable models: the Lipkin and 1D Hubbard models. The results are in general very promising. Besides the full STDDM*-b or the very related ESRPA approaches, we also investigated the solution of the one-body sector (SCRPA or CRPA) alone. Usually the results are also very good as long as one does not go with the coupling strength beyond the first phase transition point. The whole scheme can also be applied in the symmetry broken phase [25], however, the transition region needs further investigations. For practical reasons it is, of course, tempting to treat the onebody sector alone. One should, however, be aware that only STDDM*-b (and STDDM*) is a completely consistent theory when two-body correlations are incorporated. It will be very interesting to see whether STDDM*-b in the symmetry broken phase matches with the one in the unbroken phase at the transition point.

At the end, let us discuss how our formalism is related to other works. For example, there has recently been a breakthrough with the standard second RPA approach in applications to nuclei, see, e.g., [39] and references therein. Indeed, there was the longstanding problem that SRPA pushed the $p$ - $h$ states to much too low energies when coupled to the $2 p-2 h$ sector. This was experienced mostly with effective forces like Skyrme or Gogny ones. The problem came from the fact that those effective forces implicitly contain already quite some amount of higher $(m p-m h)$ correlations. The cure consists then in substracting the static part of the $2 p-2 h$ sector, so that the two-body sector completely decouples from the $p$ - $h$ sector at zero energy, see [39]. This procedure has also the appreciable property that the zero-energy spurious modes are preserved. The approach is very useful as a phenomenological procedure. The objective of the present formulation of extended second RPA is different. It aims at an $a b$ initio calculation for cases where the bare force is well known like, e.g., in Coulomb systems or, more recently, in nuclear physics where the nuclear forces are derived from chiral effective field theory. The practical advantage of the phenomenological approach is that it is formulated in the standard $p$ - $h$ and $2 p-2 h$ sub-spaces whereas the present formalism has to work in the full space in order that Goldstone theorem, sum rules, and gauge invariance remain fulfilled. This enlarges the space by a huge factor. On the other hand, as the applications show, the SCRPA sums already much of the correlations, so that the extension to second RPA can often be omitted in a first step. 
We should also mention that, contrary to the phenomenological approach where no self-consistency is involved, in our theory strong non-linearities, i.e., self-consistencies are contained, which, of course, makes the numerical problem even worse. On the other hand recently a formalism named "self-consistent configuration mixing method" was put forward [40]. There a generalised mean-field equation is solved together with the amplitudes of higher correlations and numerically applied to the ${ }^{12} \mathrm{C}$ nucleus. This method seems to demonstrate that self-consistent solutions including higher-than-one-body correlations is possible. However, the example chosen is ${ }^{12} \mathrm{C}$ with a small configuration space and a full fetched solution without truncation is still to come. Also that formalism rather is akin to what we called "odd particle number RPA" in the past [41]. In any case, we think that it is time to go beyond the standard HF-RPA approach with effective forces and to include higher correlations either into the ground state or explicitly in the form of some kind of improved second RPA.

Past collaborations on SCRPA with J. Dukelsky, D. Delion, M. Jemai are acknowledged.

\section{Appendix A. Matrices in STDDM}

The matrices $a, b, c, d$ and $\Delta d$ in eq. (22) are given below.

$$
\begin{aligned}
& a\left(\alpha \alpha^{\prime}: \lambda \lambda^{\prime}\right)=\left(\epsilon_{\alpha}-\epsilon_{\alpha^{\prime}}\right) \delta_{\alpha \lambda} \delta_{\alpha^{\prime} \lambda^{\prime}} \\
& +\sum_{\beta}\left(\bar{v}_{\alpha \lambda^{\prime} \beta \lambda} n_{\beta \alpha^{\prime}}-\bar{v}_{\beta \lambda^{\prime} \alpha^{\prime} \lambda} n_{\alpha \beta}\right), \\
& b\left(\alpha \alpha^{\prime}: \lambda_{1} \lambda_{2} \lambda_{1}^{\prime} \lambda_{2}^{\prime}\right)=\frac{1}{2}\left(\bar{v}_{\alpha \lambda_{2}^{\prime} \lambda_{1} \lambda_{2}} \delta_{\alpha^{\prime} \lambda_{1}^{\prime}}-\bar{v}_{\lambda_{1}^{\prime} \lambda_{2}^{\prime} \alpha^{\prime} \lambda_{2}} \delta_{\alpha \lambda_{1}}\right), \\
& c\left(\alpha_{1} \alpha_{2} \alpha_{1}^{\prime} \alpha_{2}^{\prime}: \lambda \lambda^{\prime}\right)=-\delta_{\alpha_{1} \lambda}\left\{\sum _ { \beta \gamma \delta } \left[\left(\delta_{\alpha_{2} \beta}-n_{\alpha_{2} \beta}\right) n_{\gamma \alpha_{1}^{\prime}} n_{\delta \alpha_{2}^{\prime}}\right.\right. \\
& \left.+n_{\alpha_{2} \beta}\left(\delta_{\gamma \alpha_{1}^{\prime}}-n_{\gamma \alpha_{1}^{\prime}}\right)\left(\delta_{\delta \alpha_{2}^{\prime}}-n_{\delta \alpha_{2}^{\prime}}\right)\right] \bar{v}_{\lambda^{\prime} \beta \gamma \delta} \\
& +\sum_{\beta \gamma}\left[\frac{1}{2} \bar{v}_{\lambda^{\prime} \alpha_{2} \beta \gamma} C_{\beta \gamma \alpha_{1}^{\prime} \alpha_{2}^{\prime}}+\bar{v}_{\lambda^{\prime} \beta \alpha_{1}^{\prime} \gamma} C_{\alpha_{2} \gamma \alpha_{2}^{\prime} \beta}\right. \\
& \left.\left.-\bar{v}_{\lambda^{\prime} \beta \alpha_{2}^{\prime} \gamma} C_{\alpha_{2} \gamma \alpha_{1}^{\prime} \beta}\right]\right\} \\
& +\delta_{\alpha_{2} \lambda}\left\{\sum _ { \beta \gamma \delta } \left[\left(\delta_{\alpha_{1} \beta}-n_{\alpha_{1} \beta}\right) n_{\gamma \alpha_{1}^{\prime}} n_{\delta \alpha_{2}^{\prime}}\right.\right. \\
& \left.+n_{\alpha_{1} \beta}\left(\delta_{\gamma \alpha_{1}^{\prime}}-n_{\gamma \alpha_{1}^{\prime}}\right)\left(\delta_{\delta \alpha_{2}^{\prime}}-n_{\delta \alpha_{2}^{\prime}}\right)\right] \bar{v}_{\lambda^{\prime} \beta \gamma \delta} \\
& +\sum_{\beta \gamma}\left[\frac{1}{2} \bar{v}_{\lambda^{\prime} \alpha_{1} \beta \gamma} C_{\beta \gamma \alpha_{1}^{\prime} \alpha_{2}^{\prime}}+\bar{v}_{\lambda^{\prime} \beta \alpha_{1}^{\prime} \gamma} C_{\alpha_{1} \gamma \alpha_{2}^{\prime} \beta}\right. \\
& \left.\left.-\bar{v}_{\lambda^{\prime} \beta \alpha_{2}^{\prime} \gamma} C_{\alpha_{1} \gamma \alpha_{1}^{\prime} \beta}\right]\right\} \\
& +\delta_{\alpha_{1}^{\prime} \lambda^{\prime}}\left\{\sum _ { \beta \gamma \delta } \left[\left(\delta_{\delta \alpha_{2}^{\prime}}-n_{\delta \alpha_{2}^{\prime}}\right) n_{\alpha_{1} \beta} n_{\alpha_{2} \gamma}\right.\right.
\end{aligned}
$$

$$
\begin{aligned}
& \left.+n_{\delta \alpha_{2}^{\prime}}\left(\delta_{\alpha_{1} \beta}-n_{\alpha_{1} \beta}\right)\left(\delta_{\alpha_{2} \gamma}-n_{\alpha_{2} \gamma}\right)\right] \bar{v}_{\beta \gamma|v| \lambda \delta} \\
& +\sum_{\beta \gamma}\left[\frac{1}{2} \bar{v}_{\beta \gamma \lambda \alpha_{2}^{\prime}} C_{\alpha_{1} \alpha_{2} \beta \gamma}+\bar{v}_{\alpha_{1} \beta \lambda \gamma} C_{\alpha_{2} \gamma \alpha_{2}^{\prime} \beta}\right. \\
& \left.\left.-\bar{v}_{\alpha_{2} \beta \lambda \gamma} C_{\alpha_{1} \gamma \alpha_{2}^{\prime} \beta}\right]\right\} \\
& -\delta_{\alpha_{2}^{\prime} \lambda^{\prime}}\left\{\sum _ { \beta \gamma \delta } \left[\left(\delta_{\delta \alpha_{1}^{\prime}}-n_{\delta \alpha_{1}^{\prime}}\right) n_{\alpha_{1} \beta} n_{\alpha_{2} \gamma}\right.\right. \\
& \left.+n_{\delta \alpha_{1}^{\prime}}\left(\delta_{\alpha_{1} \beta}-n_{\alpha_{1} \beta}\right)\left(\delta_{\alpha_{2} \gamma}-n_{\alpha_{2} \gamma}\right)\right] \bar{v}_{\beta \gamma \lambda \delta} \\
& +\sum_{\beta \gamma}\left[\frac{1}{2} \bar{v}_{\beta \gamma \lambda \alpha_{1}^{\prime}} C_{\alpha_{1} \alpha_{2} \beta \gamma}+\bar{v}_{\alpha_{1} \beta \lambda \gamma} C_{\alpha_{2} \gamma \alpha_{1}^{\prime} \beta}\right. \\
& \left.\left.-\bar{v}_{\alpha_{2} \beta \lambda \gamma} C_{\alpha_{1} \gamma \alpha_{1}^{\prime} \beta}\right]\right\} \\
& +\sum_{\beta}\left[\bar{v}_{\alpha_{1} \lambda^{\prime} \beta \lambda} C_{\beta \alpha_{2} \alpha_{1}^{\prime} \alpha_{2}^{\prime}}-\bar{v}_{\alpha_{2} \lambda^{\prime} \beta \lambda} C_{\beta \alpha_{1} \alpha_{1}^{\prime} \alpha_{2}^{\prime}}\right. \\
& \left.-\bar{v}_{\beta \lambda^{\prime} \alpha_{2}^{\prime} \lambda} C_{\alpha_{1} \alpha_{2} \alpha_{1}^{\prime} \beta}+\bar{v}_{\beta \lambda^{\prime} \alpha_{1}^{\prime} \lambda} C_{\alpha_{1} \alpha_{2} \alpha_{2}^{\prime} \beta}\right], \\
& d\left(\alpha_{1} \alpha_{2} \alpha_{1}^{\prime} \alpha_{2}^{\prime}: \lambda_{1} \lambda_{2} \lambda_{1}^{\prime} \lambda_{2}^{\prime}\right)=\left(\epsilon_{\alpha_{1}}+\epsilon_{\alpha_{2}}-\epsilon_{\alpha_{1}^{\prime}}-\epsilon_{\alpha_{2}^{\prime}}\right) \\
& \times \delta_{\alpha_{1} \lambda_{1}} \delta_{\alpha_{2} \lambda_{2}} \delta_{\alpha_{1}^{\prime} \lambda_{1}^{\prime}} \delta_{\alpha_{2}^{\prime} \lambda_{2}^{\prime}} \\
& +\frac{1}{2} \delta_{\alpha_{1}^{\prime} \lambda_{1}^{\prime}} \delta_{\alpha_{2}^{\prime} \lambda_{2}^{\prime}} \sum_{\beta \gamma}\left(\delta_{\alpha_{1} \beta} \delta_{\alpha_{2} \gamma}-\delta_{\alpha_{2} \gamma} n_{\alpha_{1} \beta}-\delta_{\alpha_{1} \beta} n_{\alpha_{2} \gamma}\right) \\
& \times \bar{v}_{\beta \gamma \lambda_{1} \lambda_{2}} \\
& -\frac{1}{2} \delta_{\alpha_{1} \lambda_{1}} \delta_{\alpha_{2} \lambda_{2}} \sum_{\beta \gamma}\left(\delta_{\alpha_{1}^{\prime} \beta} \delta_{\alpha_{2}^{\prime} \gamma}-\delta_{\alpha_{2}^{\prime} \gamma} n_{\beta \alpha_{1}^{\prime}}-\delta_{\alpha_{1}^{\prime} \beta} n_{\gamma \alpha_{2}^{\prime}}\right) \\
& \times \bar{v}_{\lambda_{1}^{\prime} \lambda_{2}^{\prime} \beta \gamma} \\
& +\delta_{\alpha_{2} \lambda_{2}} \delta_{\alpha_{2}^{\prime} \lambda_{2}^{\prime}} \sum_{\beta}\left(\bar{v}_{\alpha_{1} \lambda_{1}^{\prime} \beta \lambda_{1}} n_{\beta \alpha_{1}^{\prime}}-\bar{v}_{\beta \lambda_{1}^{\prime} \alpha_{1}^{\prime} \lambda_{1}} n_{\alpha_{1} \beta}\right) \\
& +\delta_{\alpha_{2} \lambda_{2}} \delta_{\alpha_{1}^{\prime} \lambda_{1}^{\prime}} \sum_{\beta}\left(\bar{v}_{\alpha_{1} \lambda_{2}^{\prime} \beta \lambda_{1}} n_{\beta \alpha_{2}^{\prime}}-\bar{v}_{\beta \lambda_{2}^{\prime} \alpha_{2}^{\prime} \lambda_{1}} n_{\alpha_{1} \beta}\right) \\
& +\delta_{\alpha_{1} \lambda_{1}} \delta_{\alpha_{1}^{\prime} \lambda_{1}^{\prime}} \sum_{\beta}\left(\bar{v}_{\alpha_{2} \lambda_{2}^{\prime} \beta \lambda_{2}} n_{\beta \alpha_{2}^{\prime}}-\bar{v}_{\beta \lambda_{2}^{\prime} \alpha_{2}^{\prime} \lambda_{2}} n_{\alpha_{2} \beta}\right) \\
& +\delta_{\alpha_{1} \lambda_{1}} \delta_{\alpha_{2}^{\prime} \lambda_{2}^{\prime}} \sum_{\beta}\left(\bar{v}_{\alpha_{2} \lambda_{1}^{\prime} \beta \lambda_{2}} n_{\beta \alpha_{1}^{\prime}}-\bar{v}_{\beta \lambda_{1}^{\prime} \alpha_{1}^{\prime} \lambda_{2}} n_{\alpha_{2} \beta}\right) .
\end{aligned}
$$

We now give the expression for $\Delta d$ which arises from the quadratic forms in $C_{2}$ 's of the 3-body correlation functions. We use eqs. (16) and (17) for the three-body correlation matrix;

$$
\begin{aligned}
& \Delta d\left(\alpha \beta \alpha^{\prime} \beta^{\prime}: \lambda_{1} \lambda_{2} \lambda_{1}^{\prime} \lambda_{2}^{\prime}\right)-\frac{1}{2} \bar{v}_{\alpha(h) \lambda_{1}^{\prime}(h) \lambda_{1}(p) \lambda_{2}(p)} \\
& \times C_{\lambda_{2}^{\prime}(h) \beta(h) \alpha^{\prime}(p) \beta^{\prime}(p)} \\
& +\frac{1}{2} \bar{v}_{\alpha(p) \lambda_{1}^{\prime}(p) \lambda_{1}(h) \lambda_{2}(h)} C_{\lambda_{2}^{\prime}(p) \beta(p) \alpha^{\prime}(h) \beta^{\prime}(h)} \\
& -\frac{1}{2} \delta_{\beta \lambda_{2}} \delta_{\alpha^{\prime} \lambda_{1}^{\prime}} \delta_{\beta^{\prime} \lambda_{2}^{\prime}} \sum_{\lambda(h) \lambda^{\prime}(p) \lambda^{\prime \prime}(p)} \bar{v}_{\alpha \lambda \lambda^{\prime} \lambda^{\prime \prime}} C_{\lambda^{\prime} \lambda^{\prime \prime} \lambda \lambda_{1}(h)} \\
& -\frac{1}{2} \delta_{\beta \lambda_{1}} \delta_{\alpha^{\prime} \lambda_{1}^{\prime}} \delta_{\beta^{\prime} \lambda_{2}^{\prime}} \sum_{\lambda(p) \lambda^{\prime}(h) \lambda^{\prime \prime}(h)} \bar{v}_{\alpha \lambda \lambda^{\prime} \lambda^{\prime \prime}} C_{\lambda^{\prime} \lambda^{\prime \prime} \lambda \lambda_{2}(p)}
\end{aligned}
$$




$$
\begin{aligned}
& +\frac{1}{2} \bar{v}_{\beta(h) \lambda_{1}^{\prime}(h) \lambda_{1}(p) \lambda_{2}(p)} C_{\lambda_{2}^{\prime}(h) \alpha(h) \alpha^{\prime}(p) \beta^{\prime}(p)} \\
& -\frac{1}{2} \bar{v}_{\beta(p) \lambda_{1}^{\prime}(p) \lambda_{1}(h) \lambda_{2}(h)} C_{\lambda_{2}^{\prime}(p) \alpha(p) \alpha^{\prime}(h) \beta^{\prime}(h)} \\
& +\frac{1}{2} \delta_{\alpha \lambda_{2}} \delta_{\alpha^{\prime} \lambda_{1}^{\prime}} \delta_{\beta^{\prime} \lambda_{2}^{\prime}} \sum_{\lambda(h) \lambda^{\prime}(p) \lambda^{\prime \prime}(p)} \bar{v}_{\beta \lambda \lambda^{\prime} \lambda^{\prime \prime}} C_{\lambda^{\prime} \lambda^{\prime \prime} \lambda \lambda_{1}(h)} \\
& +\frac{1}{2} \delta_{\alpha \lambda_{1}} \delta_{\alpha^{\prime} \lambda_{1}^{\prime}} \delta_{\beta^{\prime} \lambda_{2}^{\prime}} \sum_{\lambda(p) \lambda^{\prime}(h) \lambda^{\prime \prime}(h)} \bar{v}_{\beta \lambda \lambda^{\prime} \lambda^{\prime \prime}} C_{\lambda^{\prime} \lambda^{\prime \prime} \lambda \lambda_{2}(p)} \\
& +\frac{1}{2} \bar{v}_{\lambda_{1}^{\prime}(p) \lambda_{2}^{\prime}(p) \alpha^{\prime}(h) \lambda_{2}(h)} C_{\alpha(p) \beta(p) \beta^{\prime}(h) \lambda_{1}(h)} \\
& -\frac{1}{2} \bar{v}_{\lambda_{1}^{\prime}(h) \lambda_{2}^{\prime}(h) \alpha^{\prime}(p) \lambda_{2}(p)} C_{\alpha(h) \beta(h) \beta^{\prime}(p) \lambda_{1}(p)} \\
& +\frac{1}{2} \delta_{\alpha \lambda_{1}} \delta_{\beta \lambda_{2}} \delta_{\beta^{\prime} \lambda_{1}^{\prime}} \sum_{\lambda(p) \lambda^{\prime}(p) \lambda^{\prime \prime}(h)} \bar{v}_{\lambda \lambda^{\prime} \alpha^{\prime} \lambda^{\prime \prime}} C_{\lambda_{2}^{\prime}(h) \lambda^{\prime \prime} \lambda \lambda^{\prime}} \\
& -\frac{1}{2} \delta_{\alpha \lambda_{1}} \delta_{\beta \lambda_{2}} \delta_{\beta^{\prime} \lambda_{1}^{\prime}} \sum_{\lambda(h) \lambda^{\prime}(h) \lambda^{\prime \prime}(p)} \bar{v}_{\lambda \lambda^{\prime} \alpha^{\prime} \lambda^{\prime \prime}} C_{\lambda_{2}^{\prime}(p) \lambda^{\prime \prime} \lambda \lambda^{\prime}} \\
& -\frac{1}{2} \bar{v}_{\lambda_{1}^{\prime}(p) \lambda_{2}^{\prime}(p) \beta^{\prime}(h) \lambda_{2}(h)} C_{\alpha(p) \beta(p) \alpha^{\prime}(h) \lambda_{1}(h)} \\
& +\frac{1}{2} \bar{v}_{\lambda_{1}^{\prime}(h) \lambda_{2}^{\prime}(h) \beta^{\prime}(p) \lambda_{2}(p)} C_{\alpha(h) \beta(h) \alpha^{\prime}(p) \lambda_{1}(p)} \\
& -\frac{1}{2} \delta_{\alpha \lambda_{1}} \delta_{\beta \lambda_{2}} \delta_{\alpha^{\prime} \lambda_{1}^{\prime}} \sum_{\lambda(p) \lambda^{\prime}(p) \lambda^{\prime \prime}(h)} \bar{v}_{\lambda \lambda^{\prime} \beta^{\prime} \lambda^{\prime \prime}} C_{\lambda_{2}^{\prime}(h) \lambda^{\prime \prime} \lambda \lambda^{\prime}} \\
& +\frac{1}{2} \delta_{\alpha \lambda_{1}} \delta_{\beta \lambda_{2}} \delta_{\alpha^{\prime} \lambda_{1}^{\prime}} \sum_{\lambda(h) \lambda^{\prime}(h) \lambda^{\prime \prime}(p)} \bar{v}_{\lambda \lambda^{\prime} \beta^{\prime} \lambda^{\prime \prime}} C_{\lambda_{2}^{\prime}(p) \lambda^{\prime \prime} \lambda \lambda^{\prime}} .
\end{aligned}
$$

The terms with and without summation describe selfenergy corrections and vertex corrections, respectively, and indices $p(h)$ mean that the corresponding singleparticle state is a particle (hole) state.

\section{References}

1. M.P. Nightingale, C.J. Umrigar (Editors) Quantum Monte Carlo Methods in Physics and Chemistry (Springer, Berlin, 1999).

2. M. Holzmann, B. Bernu, C. Pierleoni, J. Mc Minis, D.M. Ceperly, V. Olevano, L. Delle Site, Phys. Rev. Lett. 107, 110402 (2011).

3. P.J. Knowles, C. Hampel, H.-J. Werner, J. Chem. Phys. 99, 5219 (1993).

4. R.F. Bishop, Theor. Chim. Acta 80, 95 (1991).

5. I. Peschel, X. Wang, M. Kaulke, K. Hallberg (Editors), Density-Matrix Renormalisation, A New Numerical Method in Physics (Springer, Berlin, 1999).
6. U. Schollwoeck, Ann. Phys. 326, 96 (2011).

7. U. Schollwoeck, Rev. Mod. Phys. 77, 259 (2005).

8. J.W. Clark, P. Westhaus, Phys. Rev. 141, 833 (1966).

9. G.E. Scuseria, C.L. Janssen, H.F. Schaefer, J. Chem. Phys. 89, 7382 (1988).

10. M. Tohyama, P. Schuck, Eur. Phys. J. A 50, 77 (2014).

11. S.J. Wang, W. Cassing, Ann. Phys. 159, 328 (1985).

12. P. Ring, P. Schuck, The Nuclear Many-Body Problem (Springer-Verlag, Berlin, 1980).

13. J. Dukelsky, P. Schuck, Nucl. Phys. A 512, 466 (1990).

14. P. Schuck, M. Tohyama, Phys. Rev. B 93, 165117 (2016).

15. M. Gong, M. Tohyama, Z. Phys. A 335, 153 (1990).

16. J. Dukelsky, G. Roepke, P. Schuck, Nucl. Phys. A 628, 17 (1998).

17. M. Tohyama, P. Schuck, Eur. Phys. J. A 45, 257 (2010).

18. M. Tohyama, P. Schuck, Eur. Phys. J. A 19, 215 (2004).

19. M. Tohyama, S. Takahara, P. Schuck, Eur. Phys. J. A 21, 217 (2004).

20. M. Tohyama, P. Schuck, Eur. Phys. J. A 32, 139 (2007).

21. D.J. Rowe, Rev. Mod. Phys. 40, 153 (1968).

22. D.J. Rowe, Nuclear Collective Motion, Models and Theory (World Scientific, Singapore, 2010).

23. S. Takahara, M. Tohyama, P. Schuck, Phys. Rev. C 70, 057307 (2004).

24. M. Tohyama, Phys. Rev. C 75, 044310 (2007).

25. D.S. Delion, P. Schuck, J. Dukelsky, Phys. Rev. C 72, 064305 (2005).

26. M. Kirson, Ann. Phys. 66, 624 (1971).

27. F. Catara, G. Piccitto, M. Sambataro, N. Van Giai, Phys. Rev. B 54, 17536 (1996).

28. F. Catara, M. Grasso, G. Piccitto, M. Sambataro, Phys. Rev. B 58, 16070 (1998).

29. D. Delion, P. Schuck, M. Tohyama, Eur. Phys. J. B 89, 45 (2016).

30. G. Feldman, T. Fulton, Ann. Phys. 152, 376 (1984).

31. M. Jemai, D.S. Delion, P. Schuck, Phys. Rev. C 88, 044004 (2013).

32. J.-P. Blaizot, G. Ripka, Quantum Theory of Finite Systems (The MIT Press, Cambridge, 1986).

33. J.G. Hirsch, A. Mariano, J. Dukelsky, P. Schuck, Ann. Phys. 296, 187 (2002).

34. A. Storozhenko, P. Schuck, J. Dukelsky, G. Roepke, A. Vdovin, Ann. Phys. 307, 308 (2003).

35. H.J. Lipkin, N. Meshkov, A.J. Glick, Nucl. Phys. 62, 188 (1965).

36. D. Janssen, P. Schuck, Z. Physik A 339, 43 (1991).

37. M. Jemai, P. Schuck, J. Dukelsky, R. Bennaceur, Phys. Rev. B 71, 085115 (2005).

38. M. Tohyama, Phys. Rev. C 91, 017301 (2015).

39. D. Gambacurta, M. Grasso, J. Engel, Phys. Rev. C 92, 034303 (2015).

40. C. Robin, N. Pillet, D. Pena Arteaga, J.-F. Berger, Phys. Rev. C 93, 024302 (2016).

41. M. Tohyama, P. Schuck, Phys. Rev. C 87, 044316 (2013). 